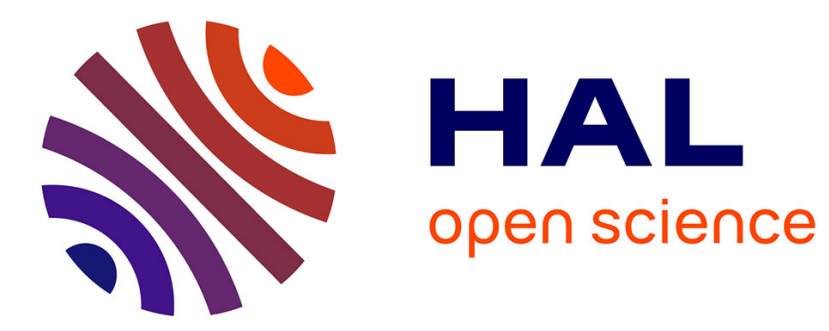

\title{
Real spectrum versus $\ell$-spectrum via Brumfiel spectrum
} Friedrich Wehrung

\section{To cite this version:}

Friedrich Wehrung. Real spectrum versus $\ell$-spectrum via Brumfiel spectrum. Algebras and Representation Theory, 2023, 26, pp.137-158. 10.1007/s10468-021-10088-0 . hal-01550450v3

\section{HAL Id: hal-01550450 \\ https://hal.science/hal-01550450v3}

Submitted on 4 Sep 2020

HAL is a multi-disciplinary open access archive for the deposit and dissemination of scientific research documents, whether they are published or not. The documents may come from teaching and research institutions in France or abroad, or from public or private research centers.
L'archive ouverte pluridisciplinaire HAL, est destinée au dépôt et à la diffusion de documents scientifiques de niveau recherche, publiés ou non, émanant des établissements d'enseignement et de recherche français ou étrangers, des laboratoires publics ou privés. 


\title{
REAL SPECTRUM VERSUS $\ell$-SPECTRUM VIA BRUMFIEL SPECTRUM
}

\author{
FRIEDRICH WEHRUNG
}

\begin{abstract}
It is well known that the real spectrum of any commutative unital ring, and the $\ell$-spectrum of any Abelian lattice-ordered group with order-unit, are all completely normal spectral spaces. We complete the existing list of containments and non-containments between the associated spectral spaces and their spectral subspaces, by proving the following results:

(1) Every real spectrum can be embedded, as a spectral subspace, into some $\ell$-spectrum.

(2) Not every real spectrum is an $\ell$-spectrum.

(3) A spectral subspace of a real spectrum may not be a real spectrum.

(4) Not every $\ell$-spectrum can be embedded, as a spectral subspace, into a real spectrum.

The commutative unital rings and Abelian lattice-ordered groups constructed in (2), (3), (4) all have cardinality $\aleph_{1}$. Moreover, (3) solves a problem stated in 2012 by Mellor and Tressl.
\end{abstract}

\section{INTRODUCTION}

Denote by $\mathbf{S X}$ the class of all spectral subspaces of members of a class $\mathbf{X}$ of spectral spaces. Most of this paper is devoted to proving the not yet established containments and non-containments, between classes of spectral spaces, represented in Figure 1.1.

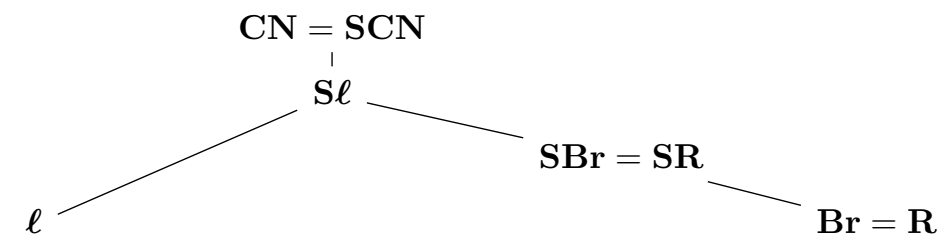

Figure 1.1. Classes of completely normal spectral spaces

The classes $\mathbf{X}$ in question are the following:

- CN, the class of all completely normal spectral spaces;

- $\ell$, the class of $\ell$-spectra of all Abelian $\ell$-groups with order-unit;

- Br, the class of Brumfiel spectra of all commutative unital $f$-rings;

- $\mathbf{R}$, the class of real spectra of all commutative unital rings.

Date: September 4, 2020

2010 Mathematics Subject Classification. 06D05; 06D50; 06F20; 03E05; 06D35.

Key words and phrases. $\ell$-group; $f$-ring; real-closed; condensate; ideal; prime; radical; spectral space; spectrum; $\ell$-spectrum; Brumfiel spectrum; real spectrum; representable; sober; Stone duality; completely normal; distributive; lattice; closed map; convex map. 
The context of our work is the following. The classical construction of the Zariski spectrum of a commutative unital ring (cf. Subsection 4.1) extends to many contexts, including distributive lattices, lattice-ordered groups ( $\ell$-groups for short), partially ordered rings, yielding Stone duality, the $\ell$-spectrum, and the real spectrum, respectively. All the topological spaces thus obtained are spectral spaces, that is, sober spaces in which the compact open subsets are a basis of the topology, closed under finite intersection. Conversely, every spectral space is the spectrum of a bounded distributive lattice (Stone [29]) and also of a commutative unital ring (Hochster [15]).

The paper will focus on the $\ell$-spectrum of an Abelian $\ell$-group (cf. Subsection 4.2) and the real spectrum of a commutative unital ring (cf. Subsection 4.4). Those two frameworks are connected by the Brumfiel spectrum of a commutative $f$-ring (cf. Subsection 4.3). All the spectral spaces thus obtained are completely normal, that is, for all elements $x$ and $y$ in the closure of a singleton $\{z\}$, either $x$ belongs to the closure of $\{y\}$ or $y$ belongs to the closure of $\{x\}$.

Prior to the present paper, part of the picture (Figure 1.1) was already known:

- Delzell and Madden [8] proved that $\ell \varsubsetneqq \mathbf{C N}$ and $\mathbf{R} \varsubsetneqq \mathbf{C N}$.

- Delzell and Madden's result got amplified in Mellor and Tressl [21], who established that any class of spectral spaces containing $\mathbf{R}$, whose Stone dual lattices are definable by a class of $\mathscr{L}_{\infty, \lambda}$-formulas for some infinite cardinal $\lambda$, has a member outside $\mathbf{S R}$. In particular, the class of all Stone duals of the spaces from $\mathbf{R}$ (resp., SR) are not $\mathscr{L}_{\infty, \lambda}$-definable. Further, $\mathbf{S R} \varsubsetneqq \mathbf{C N}$.

- Delzell and Madden [9, Proposition 3.3] observed that $\mathbf{R} \subseteq \mathbf{B r}$.

- It follows easily from Madden and Schwartz [28] and Schwartz [27] that $\mathbf{B r} \subseteq \mathbf{R}$. Consequently, $\mathbf{B r}=\mathbf{R}$ (cf. Corollary 4.17).

- The author proved in [32] that every second countable completely normal spectral space is in $\boldsymbol{\ell}$, and that moreover, the class of all Stone duals of spaces from $\boldsymbol{\ell}$ is not $\mathscr{L}_{\infty, \omega}$-definable.

- The author provided an example in [32] showing that $\boldsymbol{\ell} \varsubsetneqq \mathbf{S} \boldsymbol{\ell}$.

- The present paper's first version, available on the HAL preprint server since 2017 , established, with a counterexample with $\aleph_{2}$ compact open subsets, that $\mathbf{S} \boldsymbol{\ell} \varsubsetneqq \mathbf{C N}$. In the meantime, the preprint got lost in an editorial process and the result got superseded by the author's more recent paper [34], which provided a stronger counterexample. For more details see Section 7 .

The missing pieces, all provided in the present paper, are the following:

- It is not hard to verify that every Brumfiel spectrum, thus also every real spectrum, can be embedded, as a spectral subspace, into some $\ell$-spectrum. This is stated in Corollary 5.7. Hence, $\mathbf{S B r} \subseteq \mathbf{S} \boldsymbol{\ell}$.

- Not every real spectrum is an $\ell$-spectrum. This is established in Theorem 5.4, via the construction of a condensate. Hence, $\mathbf{R} \nsubseteq \boldsymbol{\ell}$.

- A spectral subspace of a real spectrum may not be a Brumfiel spectrum (thus also not a real spectrum). This is stated in Corollary 5.10, via the construction of a condensate. It follows that $\mathbf{S R} \nsubseteq \mathbf{B r}$. This solves a problem of Mellor and Tressl [21]. 
- Not every $\ell$-spectrum can be embedded, as a spectral subspace, into a Brumfiel spectrum (thus also not into a real spectrum). Hence, $\ell \nsubseteq \mathbf{S B r}$. This is stated in Corollary 6.8.

We point out that although not every $\ell$-spectrum is a real spectrum (cf. Corollary 6.8), there is a formally related problem, on the interaction between Abelian $\ell$-groups and commutative rings, with a well known positive solution. Let $D$ be an integral domain with group of units $U$ and field of fractions $K$. Denote by $K^{\times}$the multiplicative group of all nonzero elements of $K$. The group of divisibility of $D$ (cf. Močkoř [23]) is the quotient group $K^{\times} / U$, endowed with the unique translationinvariant partial ordering with positive cone $D / U$. Every Abelian $\ell$-group is the group of divisibility of some integral domain, which, in addition, can be taken a Bezout domain (cf. Anderson [1, page 4], where the result is credited to Krull, Jaffard, Kaplansky, and Ohm). Thus, Corollary 6.8 illustrates the gap between the group of divisibility and the real spectrum.

Since Stone duality is more conveniently stated with bounded distributive lattices, our results on spectral spaces are mostly formulated for commutative, unital rings and Abelian $\ell$-groups with order-unit. On the other hand, most of our latticetheoretical results are valid for lattices without top element, and thus formulated in that more general context.

Methodology. The underlying counterexamples of Theorems 5.4 and 5.9 are constructed with the one-arrow version, originating in the author's paper [31], of the general categorical model-theoretical construction of condensates developed in Gillibert and Wehrung [13]. A condensate of a diagram $\vec{A}$ is a special kind of directed colimit of finite products of vertices of $\vec{A}$. The condensate construction makes it possible to turn diagram counterexamples, to representation problems with respect to a given functor, to object counterexamples. In the one-arrow case required here, non-representable arrows are turned to non-representable objects, via the simplified condensate construction described in Definition 5.1 together with Lemma 5.3 (getting non- $\ell$-representable distributive lattices) and its variant Lemma 5.8 (getting non-Brumfiel-representable distributive lattices).

In particular, the proof of Theorem 5.4, obtained by applying the condensate construction to an embedding $A \hookrightarrow K$ of countable real-closed rings, produces a real-closed ring of cardinality $\aleph_{1}$. Changing context, the proof of Theorem 5.9, obtained by applying the condensate construction to a surjective homomorphism between finite chains, produces a real spectrum, with $\aleph_{1}$ compact open subsets, with a spectral subspace which is not a real spectrum.

On the other hand, Theorem 6.4, producing the $\ell$-spectrum of a unital Abelian $\ell$-group of cardinality $\aleph_{1}$ which cannot be embedded as a spectral subspace into any real spectrum, is established via a direct argument, centered on the lexicographical product of a restricted Hahn power of the integers by the free Abelian $\ell$-group on two positive generators.

\section{BASIC CONCEPTS}

For $\ell$-groups and $f$-rings, we refer the reader to Bigard, Keimel, and Wolfenstein [6] or Anderson and Feil [2]. For any partially ordered Abelian group $G$, we set $G^{+} \stackrel{\text { def }}{=}\{x \in G \mid x \geq 0\}$ (the positive cone of $G$ ) and $G^{++} \stackrel{\text { def }}{=}\{x \in G \mid x>0\}$. For $a, b \in G^{+}$, let $a \ll b$ hold if $k a \leq b$ for every positive integer $k$. 
For partially ordered Abelian groups $G$ and $H$, the lexicographical product of $G$ by $H$, denoted $G \times \times_{\text {lex }} H$, is the product group $G \times H$, endowed with the positive cone consisting of all pairs $(x, y)$ with either $x>0$ or $(x=0$ and $y \geq 0)$.

For any chain $\Lambda$, we denote by $\mathbb{Z}\langle\Lambda\rangle$ the restricted lexicographical power, of the chain $\mathbb{Z}$ of all integers, by $\Lambda$. Hence the elements of $\mathbb{Z}\langle\Lambda\rangle$ have the form $x=\sum_{i=1}^{n} k_{i} c_{\xi_{i}}$, where each $k_{i} \in \mathbb{Z} \backslash\{0\}$ and $\xi_{1}<\cdots<\xi_{n}$ in $\Lambda$, and $x$ belongs to the positive cone of $\mathbb{Z}\langle\Lambda\rangle$ iff either $n=0$ (i.e., $x=0$ ) or $k_{n}>0$. This endows $\mathbb{Z}\langle\Lambda\rangle$ with a structure of a totally ordered Abelian group.

A lattice-ordered group, or $\ell$-group for short, is a group endowed with a translation-invariant lattice ordering. All our $\ell$-groups will be Abelian and will thus be denoted additively. Elements $x$ and $y$, in an $\ell$-group, are orthogonal if $x \wedge y=0$.

A subset $I$ in an Abelian $\ell$-group $G$ is an $\ell$-ideal if it simultaneously a subgroup of $G$ and an order-convex sublattice of $G$.

For any elements $a$ and $b$ in an Abelian $\ell$-group $G$, we will set $a^{+} \stackrel{\text { def }}{=} a \vee 0$, $a^{-} \stackrel{\text { def }}{=}(-a) \vee 0,|a| \stackrel{\text { def }}{=} a \vee(-a)$, and $a \backslash b \stackrel{\text { def }}{=}(a-b)^{+}=a-(a \wedge b)$.

A lattice-ordered ring is a ring endowed with a lattice ordering invariant under additive translations and preserved by multiplicative translations by positive elements. A lattice-ordered $\operatorname{ring} A$ is an $f$-ring if $x \wedge y=0$ implies that $x \wedge y z=$ $x \wedge z y=0$ whenever $x, y, z \in A^{+}$and $x \wedge y=0$. Equivalently, $A$ is a subdirect product of totally ordered (not necessarily unital) rings (cf. Bigard, Keimel, and Wolfenstein [6, Théorème 9.1.2]).

Lemma 2.1 (folklore). Let $G$ be an Abelian $\ell$-group and let $a, b, c \in G$. Then

- $a \backslash c \leq(a \backslash b)+(b \backslash c)$.

- $(a \backslash b) \wedge(b \backslash a)=0$.

- If, in addition, $G$ is the underlying additive $\ell$-group of an $f$-ring $A$ and $c \in A^{+}$, then $c a \backslash c b=c(a \backslash b)$ and $a c \backslash b c=(a \backslash b) c$.

A subset $I$ in an $f$-ring $A$ is an $\ell$-ideal if it is, simultaneously, an ideal of the underlying ring of $A$ and an order-convex sublattice of $A$.

Totally ordered rings are particular cases of $f$-rings. About those, we will need the following lemma.

Lemma 2.2. Let $A$ be a totally ordered (not necessarily unital) commutative domain and let $I$ be a proper order-convex ideal of $A$. Then for every $x \in I$ and every $a \in A$, the relation $|x a| \ll|a|$ holds.

Proof. We will use repeatedly the fact that for every $c \in A^{++}$, the assignment $t \mapsto t c$ defines an order-embedding of $A$ into itself. Since $A$ is totally ordered, we may assume that $a \geq 0$ and $x \geq 0$. Let $n<\omega$ and suppose that $n x a>a$ (so $a>0$ ). Then for every $b \in A^{+}, n x a b \geq a b \geq 0$, thus (as $a>0$ ) $n x b \geq b \geq 0$. Since $x \in I$ and $I$ is an ideal of $A$, we get $n x b \in I$. Since $I$ is order-convex, it follows that $b \in I$. This holds for every $b \in A^{+}$, whence $I=A$, a contradiction. Since $A$ is totally ordered, it follows that $n x a \leq a$.

\section{Stone DUALity BetWeEn Distributive LATTICES With ZERO AND GENERALIZED SPECTRAL SPACES}

For lattice theory we refer the reader to Grätzer [14], Johnstone [17]. For any elements $a$ and $b$ in a distributive lattice $D$ with zero, a splitting of $(a, b)$ is a pair $(x, y)$ of elements of $D$ such that $a \vee b=a \vee y=x \vee b$ and $x \wedge y=0$. Observe 
that in that case, $x \leq a$ and $y \leq b$. We say that $D$ is completely normal if every pair of elements in $D$ has a splitting.

We denote by $P^{\text {op }}$ the opposite poset of a poset $P$. For any functions $f$ and $g$ with common domain $X$, we set

$$
\llbracket f \neq g \rrbracket \stackrel{\text { def }}{=}\{x \in X \mid f(x) \neq g(x)\} .
$$

For posets $P$ and $Q$, a map $f: P \rightarrow Q$ is cofinal if every element of $Q$ lies below some element of the range of $f$.

Definition 3.1. For a topological space $X$, we denote by $\stackrel{\circ}{\mathcal{K}}(X)$ the set of all compact ${ }^{1}$ open subsets of $X$, ordered under set inclusion. We say that $X$ is

- sober, if every join-irreducible member, of the lattice of all closed subsets of $X$, is the closure of a unique singleton ${ }^{2}$;

- generalized spectral, if it is sober, $\mathcal{K}(X)$ is a basis of the topology of $X$, and $U \cap V$ is compact whenever $U$ and $V$ are compact open subsets of $X$;

- spectral, if it is simultaneously compact and generalized spectral.

The specialization preorder on $X$ is defined by

$$
x \leqslant y \text { if } y \in \operatorname{cl}_{X}(\{x\}), \quad \text { for all } x, y \in X .
$$

A map $\varphi: X \rightarrow Y$, between generalized spectral spaces, is spectral if the inverse image under $\varphi$, of any compact open subset of $Y$, is a compact open subset of $X$.

The spectrum $\operatorname{Spec} D$, of a distributive lattice $D$ with zero, is defined as the set of all (proper) prime ideals of $D$, endowed with the closed sets $\{P \in \operatorname{Spec} D \mid I \subseteq P\}$, for subsets (equivalently, ideals) $I$ of $D$. The specialization order on $\operatorname{Spec} D$ is just set-theoretical inclusion. The assignments $D \mapsto \operatorname{Spec} D, X \mapsto \stackrel{\circ}{\mathcal{K}}(X)$ can be naturally extended to morphisms, yielding Stone duality (cf. Stone [29], Johnstone [17, § II.3], Grätzer [14, § II.5], and Rump and Yang [25, page 63] for the general, non necessarily unital case) between distributive lattices with zero with cofinal 0-lattice homomorphisms and generalized spectral spaces with spectral maps.

Remark 3.2. Let $X$ and $Y$ be generalized spectral spaces, with $X$ contained (in the set-theoretical sense) in $Y$, and let $\varphi: X \hookrightarrow Y$ be the inclusion map. We say that $X$ is a spectral subspace of $Y$ if the topology of $X$ is the topology induced by the topology of $Y$ and the map $\varphi$ is spectral. In that case, the dual map $\stackrel{\circ}{\mathcal{K}}(\varphi): \stackrel{\circ}{\mathcal{K}}(Y) \rightarrow$ $\stackrel{\circ}{\mathcal{K}}(X), V \mapsto X \cap V$ is a surjective lattice homomorphism. Conversely, for every surjective lattice homomorphism $f: D \rightarrow E$, the spectral map Spec $f: \operatorname{Spec} E \rightarrow$ $\operatorname{Spec} D$ is a spectral embedding, that is, it embeds $\operatorname{Spec} E$ into $\operatorname{Spec} D$ as a spectral subspace. Hence, spectral subspaces correspond, via Stone duality, to surjective lattice homomorphisms.

The generalized spectral spaces $X$ that we will consider in this paper will mostly be completely normal. By Monteiro [22, Théorème V.3.1], this is equivalent to saying that the dual lattice $\stackrel{\circ}{\mathcal{K}}(X)$ is completely normal (cf. Section 2 for the definition of completely normal lattices).

\footnotetext{
${ }^{1}$ Throughout the paper, "compact" means what some other references call "quasicompact"; in particular, it does not imply Hausdorff.

${ }^{2}$ Due to the uniqueness, every sober space is $\mathrm{T}_{0}$ (not all references assume this).
} 
We denote by $\mathfrak{P}(X)$ the powerset of any set $X$, ordered under set inclusion. We denote by $\omega_{\alpha}$, or $\aleph_{\alpha}$ according to the context ("ordinal versus cardinal"), the $\alpha$ th infinite cardinal, and we set $\omega \stackrel{\text { def }}{=} \omega_{0}=\{0,1,2, \ldots\}$.

Throughout the paper, "countable" means "at most countable".

\section{ZARISKI, $\ell$, BRUMFIEL, REAL: SPECTRA AND LATTICES}

In this section we recall some well known facts on the various sorts of spectra and distributive lattices that will intervene in the paper. We also introduce some notation and include a few new results, such as Lemma 4.11. For more details and references, we refer the reader to Delzell and Madden [9], Johnstone [17, Chapter 5], Keimel [19], Coste and Roy [7], Dickmann [10, Chapter 6].

4.1. Zariski spectrum. The (Zariski) spectrum of a commutative unital ring $A$ is defined as the set Spec $A$ of all prime ideals of $A$, endowed with the topology whose closed sets are exactly the sets $\operatorname{Spec}(A, I) \stackrel{\text { def }}{=}\{P \in \operatorname{Spec} A \mid I \subseteq P\}$, for subsets (equivalently, radical ideals) $I$ of $A$.

Denote by $\left\langle a_{1}, \ldots, a_{m}\right\rangle^{\mathrm{r}}$ the radical ideal of $A$ generated by elements $a_{1}, \ldots$, $a_{m}$ of $A$, and denote ${ }^{3}$ by $\operatorname{Id}_{\mathrm{c}}^{\mathrm{r}} A$ the set of all ideals of $A$ of the form $\left\langle a_{1}, \ldots, a_{m}\right\rangle^{\mathrm{r}}$ (finitely generated radical ideals), ordered by set inclusion. Due to the formulas

$$
\begin{aligned}
& \left\langle a_{1}, \ldots, a_{m}\right\rangle^{\mathrm{r}} \vee\left\langle b_{1}, \ldots, b_{n}\right\rangle^{\mathrm{r}}=\left\langle a_{1}, \ldots, a_{m}, b_{1}, \ldots, b_{n}\right\rangle^{\mathrm{r}}, \\
& \left.\left\langle a_{1}, \ldots, a_{m}\right\rangle^{\mathrm{r}} \cap\left\langle b_{1}, \ldots, b_{n}\right\rangle^{\mathrm{r}}=\left\langle a_{i} b_{j}\right| 1 \leq i \leq m \text { and } 1 \leq j \leq n\right\rangle^{\mathrm{r}},
\end{aligned}
$$

(where $\vee$ stands for the join in the lattice of all radical ideals of $A$ ), $\operatorname{Id}_{\mathrm{c}}^{\mathrm{r}} A$ is a 0 -sublattice of the distributive lattice of all radical ideals of $A$.

Since every radical ideal of $A$ is the intersection of all prime ideals containing it, $\operatorname{Id}_{\mathrm{c}}^{\mathrm{r}} A$ is the Stone dual of Spec $A$ (cf. Delzell and Madden [9, page 115]):

Proposition 4.1. The Zariski spectrum $\operatorname{Spec} A$, of a commutative unital ring A, is a spectral space, and the assignment $I \mapsto\{P \in \operatorname{Spec} A \mid I \nsubseteq P\}$ defines an isomorphism from $\operatorname{Id}_{\mathrm{c}}^{\mathrm{r}} A$ onto the Stone dual $\stackrel{\circ}{\mathcal{K}}(\operatorname{Spec} A)$ of $\operatorname{Spec} A$.

Due to the following deep result by Hochster [15], there is no need to imagine a new name for the class of all lattices of the form $\operatorname{Id}_{\mathrm{c}}^{\mathrm{r}} A$.

Theorem 4.2 (Hochster). Every spectral space is homeomorphic to the Zariski spectrum of some commutative unital ring. Hence, every bounded distributive lattice is isomorphic to $\mathrm{Id}_{\mathrm{c}}^{\mathrm{r}}$ A for some commutative unital ring $A$.

4.2. $\ell$-spectrum and $\ell$-representable lattices. The $\ell$-spectrum of an Abelian $\ell$-group $G$ is defined as the set $\operatorname{Spec}_{\ell} G$ of all prime $\ell$-ideals of $G$, endowed with the topology whose closed sets are exactly the $\left\{P \in \operatorname{Spec}_{\ell} G \mid I \subseteq P\right\}$, for subsets (equivalently, $\ell$-ideals) $I$ of $G$.

Denote by $\left\langle a_{1}, \ldots, a_{m}\right\rangle^{\ell}$, or $\left\langle a_{1}, \ldots, a_{m}\right\rangle_{G}^{\ell}$ if $G$ needs to be specified, the $\ell$-ideal of $G$ generated by elements $a_{1}, \ldots, a_{m}$ of $G$, and denote by $\operatorname{Id}_{\mathrm{c}}^{\ell} G$ the set of all $\ell$-ideals of $G$ of the form $\left\langle a_{1}, \ldots, a_{m}\right\rangle^{\mathrm{r}}$ (finitely generated $\ell$-ideals), ordered by

\footnotetext{
${ }^{3}$ The subscript "c" stands for "compact", which is the lattice-theoretical formalization of "finitely generated". The superscript "r" stands for "radical".
} 
set inclusion. Since $\left\langle a_{1}, \ldots, a_{m}\right\rangle^{\ell}=\langle a\rangle^{\ell}$ where $a \stackrel{\text { def }}{=} \sum_{i=1}^{m}\left|a_{i}\right|$, we get $\operatorname{Id}_{\mathrm{c}}^{\ell} G=$ $\left\{\langle a\rangle^{\ell} \mid a \in G^{+}\right\}$. Due to the formulas

$$
\langle a\rangle^{\ell} \vee\langle b\rangle^{\ell}=\langle a+b\rangle^{\ell} \text { and }\langle a\rangle^{\ell} \cap\langle b\rangle^{\ell}=\langle a \wedge b\rangle^{\ell}, \quad \text { for all } a, b \in G^{+}
$$

(where $\vee$ stands for the join in the lattice of all $\ell$-ideals of $A$ ), $\operatorname{Id}_{\mathrm{c}}^{\ell} G$ is a 0 -sublattice of the distributive lattice of all $\ell$-ideals of $G$. It has a top element iff $G$ has an orderunit.

Since every $\ell$-ideal of $G$ is the intersection of all prime $\ell$-ideals containing it, $\operatorname{Id}_{\mathrm{c}}^{\mathrm{r}} G$ is the Stone dual of $\operatorname{Spec}_{\ell} G$ (cf. Proposition 1.19, together with Theorem 1.10 and Lemma 1.20, in Keimel [18]):

Proposition 4.3. The $\ell$-spectrum $\operatorname{Spec}_{\ell} G$, of any Abelian $\ell$-group $G$, is a generalized spectral space, and the assignment $I \mapsto\{P \in \operatorname{Spec} G \mid I \nsubseteq P\}$ defines an isomorphism from $\operatorname{Id}_{\mathrm{c}}^{\ell} G$ onto the Stone dual $\stackrel{\circ}{\mathcal{K}}\left(\operatorname{Spec}_{\ell} G\right)$ of $\operatorname{Spec}_{\ell} G$.

Following terminology from Iberkleid, Martínez, and McGovern [16] and Wehrung [32], we recall the following definition.

Definition 4.4. For distributive lattices $A$ and $B$, a map $f: A \rightarrow B$ is closed if for all $a_{0}, a_{1} \in A$ and all $b \in B$, if $f\left(a_{0}\right) \leq f\left(a_{1}\right) \vee b$, then there exists $x \in A$ such that $a_{0} \leq a_{1} \vee x$ and $f(x) \leq b$.

The following lemma is established in Wehrung [32].

Lemma 4.5. Let $A$ and $B$ be Abelian $\ell$-groups and let $f: A \rightarrow B$ be an $\ell$-homomorphism. Then the map $\operatorname{Id}_{\mathrm{c}}^{\ell} f: \operatorname{Id}_{\mathrm{c}}^{\ell} A \rightarrow \operatorname{Id}_{\mathrm{c}}^{\ell} B,\langle x\rangle^{\ell} \mapsto\langle f(x)\rangle^{\ell}$ is a closed 0-lattice homomorphism.

In particular, the assignments $G \mapsto \operatorname{Id}_{\mathrm{c}}^{\ell} G, f \mapsto \operatorname{Id}_{\mathrm{c}}^{\ell} f$ define a functor, from the category of all Abelian $\ell$-groups with $\ell$-homomorphisms, to the category of all distributive lattices with zero with closed 0-lattice homomorphisms. It is well known that this functor preserves nonempty finite direct products and directed colimits.

Say that a lattice $D$ is $\ell$-representable if it is isomorphic to $\operatorname{Id}_{\mathrm{c}}^{\ell} G$ for some Abelian $\ell$-group $G$. Equivalently, the spectrum of $D$ is homeomorphic to the $\ell$-spectrum of some Abelian $\ell$-group. This terminology is extended to diagrams $\vec{D}$ of distributive lattices with zero and 0-lattice homomorphisms, by saying that $\vec{D} \cong \operatorname{Id}_{\mathrm{c}}^{\ell} \vec{G}$ for some diagram $\vec{G}$ of Abelian $\ell$-groups and $\ell$-homomorphisms.

It is well known that every $\ell$-representable lattice is completely normal. The author established the following result in [32].

Theorem 4.6. Every countable completely normal distributive lattice with zero is $\ell$-representable. On the other hand, the class of all $\ell$-representable lattices cannot be defined by a class of $\mathscr{L}_{\infty, \omega}$-formulas of lattice theory.

The following easy lemma is established in Wehrung [32].

Lemma 4.7. Let $G$ be an Abelian $\ell$-group, let $S$ be a distributive lattice with zero, and let $\varphi: \operatorname{Id}_{\mathrm{c}}^{\ell} G \rightarrow S$ be a closed surjective 0-lattice homomorphism. Then $I \stackrel{\text { def }}{=}\left\{x \in G \mid \varphi\left(\langle x\rangle^{\ell}\right)=0\right\}$ is an $\ell$-ideal of $G$, and there is a unique isomorphism $\psi: \operatorname{Id}_{\mathrm{c}}^{\ell}(G / I) \rightarrow S$ such that $\psi\left(\langle x+I\rangle^{\ell}\right)=\varphi\left(\langle x\rangle^{\ell}\right)$ for every $x \in G^{+}$. 
4.3. Brumfiel spectrum and Brumfiel-representable lattices. For any commutative $f$-ring $A$, we say that a (proper) $\ell$-ideal $P$ is prime if it is both an $\ell$-ideal and prime as a ring ideal. Then $P$ is also prime as an $\ell$-ideal of the underlying additive $\ell$-group of $A$, that is, $x \wedge y \in P$ implies that either $x \in P$ or $y \in P$, whenever $x, y \in A$.

The Brumfiel spectrum of a commutative $f$-ring $A$ is defined as the set $\operatorname{Spec}_{\mathrm{B}} A$ of all prime $\ell$-ideals of $A$, endowed with the topology whose closed sets are exactly the $\left\{P \in \operatorname{Spec}_{\mathrm{B}} A \mid I \subseteq P\right\}$, for subsets (equivalently, radical $\ell$-ideals) $I$ of $A$.

Denote by $\left\langle a_{1}, \ldots, a_{m}\right\rangle^{\mathrm{r}}$, or $\left\langle a_{1}, \ldots, a_{m}\right\rangle_{A}^{\mathrm{r}}$ if $A$ needs to be specified, the radical $\ell$-ideal of $A$ generated by elements $a_{1}, \ldots, a_{m}$ of $A$, and denote by $\operatorname{Id}_{\mathrm{c}}^{\mathrm{r}} A$ the set of all ideals of $A$ of the form $\left\langle a_{1}, \ldots, a_{m}\right\rangle^{\mathrm{r}}$ (finitely generated radical ideals), ${ }^{4}$ ordered by set inclusion. Since $\left\langle a_{1}, \ldots, a_{m}\right\rangle^{\mathrm{r}}=\langle a\rangle^{\ell}$ where $a \stackrel{\text { def }}{=} \sum_{i=1}^{m}\left|a_{i}\right|$, we get $\operatorname{Id}_{\mathrm{c}}^{\mathrm{r}} A=\left\{\langle a\rangle^{\mathrm{r}} \mid a \in A^{+}\right\}$. Due to the formulas

$$
\langle a\rangle^{\mathrm{r}} \vee\langle b\rangle^{\mathrm{r}}=\langle|a|+|b|\rangle^{\mathrm{r}} \text { and }\langle a\rangle^{\mathrm{r}} \cap\langle b\rangle^{\mathrm{r}}=\langle|a| \wedge|b|\rangle^{\mathrm{r}}=\langle a b\rangle^{\mathrm{r}}, \quad \text { for all } a, b \in A
$$

(where $\vee$ stands for the join in the lattice of all radical $\ell$-ideals of $A$ ), $\operatorname{Id}_{\mathrm{c}}^{\mathrm{r}} A$ is a 0 -sublattice of the distributive lattice of all radical $\ell$-ideals of $A$. If $A$ is unital, then $\operatorname{Id}_{\mathrm{c}}^{\mathrm{r}} A$ has a top element.

Since every radical $\ell$-ideal of $A$ is the intersection of all prime $\ell$-ideals containing it, $\operatorname{Id}_{\mathrm{c}}^{\mathrm{r}} A$ is the Stone dual of $\operatorname{Spec}_{\mathrm{B}} A$ (cf. Delzell and Madden [9, Proposition 4.2]):

Proposition 4.8. The Brumfiel spectrum $\operatorname{Spec}_{\mathrm{B}} A$, of a commutative $f$-ring $A$, is a generalized spectral space, and the assignment $I \mapsto\left\{P \in \operatorname{Spec}_{\mathrm{B}} A \mid I \nsubseteq P\right\}$ defines an isomorphism from $\operatorname{Id}_{\mathrm{c}}^{\mathrm{r}} A$ onto the Stone dual $\stackrel{\circ}{\mathcal{K}}\left(\operatorname{Spec}_{\mathrm{B}} A\right)$ of $\operatorname{Spec}_{\mathrm{B}} A$.

Say that a lattice $D$ is Brumfiel-representable if it is isomorphic to $\operatorname{Id}_{\mathrm{c}}^{\mathrm{r}} A$ for some commutative $f$-ring $A$. Equivalently, the spectrum of $D$ is homeomorphic to the Brumfiel spectrum of some commutative $f$-ring. As in Subsection 4.2, this terminology is extended to diagrams of lattices, in a standard fashion.

It is well known that every Brumfiel-representable lattice is completely normal. We will see, with Corollary 6.8 in the present paper, that not every $\ell$-representable lattice (thus, a fortiori, not every completely normal distributive lattice) is Brumfiel-representable.

The following result is an analogue of Lemma 4.7 for $f$-rings. Its proof is similar and we omit it.

Lemma 4.9. Let $A$ be a commutative f-ring, let $S$ be a distributive lattice with zero, and let $\varphi: \operatorname{Id}_{\mathrm{c}}^{\mathrm{r}} A \rightarrow S$ be a closed surjective 0-lattice homomorphism. Then $I \stackrel{\text { def }}{=}$ $\left\{x \in A \mid \varphi\left(\langle x\rangle^{\mathrm{r}}\right)=0\right\}$ is a radical $\ell$-ideal of $A$, and there is a unique isomorphism $\psi: \operatorname{Id}_{\mathrm{c}}^{\mathrm{r}}(A / I) \rightarrow S$ such that $\psi\left(\langle x+I\rangle^{\mathrm{r}}\right)=\varphi\left(\langle x\rangle^{\mathrm{r}}\right)$ for every $x \in A^{+}$.

Definition 4.10. Let $A$ and $B$ be distributive lattices with zero. A 0-lattice homomorphism $f: A \rightarrow B$ is convex if for all $P \in \operatorname{Spec} A$ and all $Q_{0}, Q_{1} \in \operatorname{Spec} B$, if $Q_{0} \subseteq Q_{1}$ and $f^{-1}\left[Q_{0}\right] \subseteq P \subseteq f^{-1}\left[Q_{1}\right]$, then there exists $Q \in \operatorname{Spec} B$ such that $Q_{0} \subseteq Q \subseteq Q_{1}$ and $P=f^{-1}[Q]$.

Equivalently, the Stone dual map $\operatorname{Spec} f: \operatorname{Spec} B \rightarrow \operatorname{Spec} A$ is convex in the sense of Dickmann, Schwartz, and Tressl [12, Definition 13.3.10].

\footnotetext{
${ }^{4}$ Although we are using, for radical $\ell$-ideals, the same notation as the one in Subsection 4.1 for radical ideals, the context will always make it clear which concept is used.
} 
The following result extends to the Brumfiel spectrum functor a result originally established for the real spectrum functor in Korollar 4, pages 133-134 of Knebusch and Scheiderer [20]. Our proof is a straightforward modification of its analogue for real spectra, stated in the monograph Dickmann, Schwartz, and Tressl [12, Theorem 13.3.12]. We include it for convenience.

Lemma 4.11. Let $A$ and $B$ be commutative f-rings and let $f: A \rightarrow B$ be a homomorphism of $f$-rings. Then the map $\operatorname{Id}_{\mathrm{c}}^{\mathrm{r}} f: \operatorname{Id}_{\mathrm{c}}^{\mathrm{r}} A \rightarrow \operatorname{Id}_{\mathrm{c}}^{\mathrm{r}} B$ is convex.

Proof. By Stone duality, it is sufficient to prove that for all $P \in \operatorname{Spec}_{\mathrm{B}} A$ and all $Q_{0}, Q_{1} \in \operatorname{Spec}_{\mathrm{B}} B$, if $Q_{0} \subseteq Q_{1}$ and $f^{-1}\left[Q_{0}\right] \subseteq P \subseteq f^{-1}\left[Q_{1}\right]$, then there exists $Q \in \operatorname{Spec}_{\mathrm{B}} B$ such that $Q_{0} \subseteq Q \subseteq Q_{1}$ and $P=f^{-1}[Q]$. We may replace $A$ by $A / f^{-1}\left[Q_{0}\right], B$ by $B / Q_{0}, Q_{1}$ by $Q_{1} / Q_{0}$, and $f$ by the canonical embedding $A / f^{-1}\left[Q_{0}\right] \rightarrow B / Q_{0}$. Hence, we may assume that $A$ is an ordered subring of a totally ordered (not necessarily unital) commutative domain $B, Q_{0}=\{0\}, f$ is the inclusion map from $A$ into $B, P \in \operatorname{Spec}_{\mathrm{B}} A, Q_{1} \in \operatorname{Spec}_{\mathrm{B}} B$, and $P \subseteq Q_{1}$. We must find $Q \in \operatorname{Spec}_{\mathrm{B}} B$ such that $P=Q \cap A$ and $Q \subseteq Q_{1}$. We set

$$
Q \stackrel{\text { def }}{=}\left\{y \in Q_{1} \mid(\exists n \in \omega \backslash\{0\})(\exists x \in P)\left(|y|^{n} \leq x\right)\right\} .
$$

We claim that $Q$ is a prime $\ell$-ideal of $B$. It is obvious that $Q$ is an order-convex $\ell$-subgroup of $B$. Now let $y \in Q$ and $b \in B$. We must prove that $y b \in Q$. Since $B$ is totally ordered, we may assume that $\{y, b\} \subseteq B^{+}$. Since $Q_{1}$ is an ideal of $B$, $y b \in Q_{1}$. By assumption, there are $n \in \omega \backslash\{0\}$ and $x \in P$ such that $y^{n} \leq x$. It follows that $y^{n} \in Q_{1}$, thus, since $Q_{1}$ is a radical ideal of $B, y \in Q_{1}$, and thus $y b^{n+1} \in Q_{1}$. Since $Q_{1}$ is a proper $\ell$-ideal of $B$, it follows, using Lemma 2.2, that $(y b)^{n+1}=y^{n}\left(y b^{n+1}\right) \leq y^{n} \leq x$, whence $y b \in Q$. This completes the proof that $Q$ is an $\ell$-ideal of $B$.

Let $x, y \in B$ such that $x y \in Q$, we must prove that $x \in Q$ or $y \in Q$. Since $B$ is totally ordered, we may assume that $0 \leq x \leq y$. There are $n \in \omega \backslash\{0\}$ and $p \in P$ such that $(x y)^{n} \leq p$. It follows that $x^{2 n} \leq(x y)^{n} \leq p$, whence $x \in Q$, thus completing the proof that $Q$ is prime.

Now it is obvious that $Q \subseteq Q_{1}$ and $P=Q \cap A$.

4.4. Real spectrum and real-representable lattices. Let $A$ be a commutative unital ring. A subset $C$ of $A$ is a cone if it is both an additive and a multiplicative submonoid of $A$, containing all squares in $A$. A cone $P$ of $A$ is prime if $A=$ $P \cup(-P)$ and the support $P \cap(-P)$ is a prime ideal of $A$. For a prime cone $P$, $-1 \notin P$ (otherwise $1 \in P \cap(-P)$, thus $P \cap(-P)=A$, a contradiction). We denote by $\operatorname{Spec}_{\mathrm{r}} A$ the set of all prime cones of $A$, endowed with the topology generated by all subsets of the form $\left\{P \in \operatorname{Spec}_{\mathrm{r}} A \mid a \notin P\right\}$, for $a \in A$, and we call $\operatorname{Spec}_{\mathrm{r}} A$ the real spectrum of $A$.

It is not so straightforward to describe directly the Stone dual of $\mathrm{Spec}_{\mathrm{r}} A$. However, it is possible to reduce it to the Brumfiel spectrum, as follows. The universal f-ring $\mathrm{F}(A)$ of $A$ is a commutative unital $f$-ring. The first statement in the following result is established in Delzell and Madden [9, Proposition 3.3]. The second statement follows by using Proposition 4.8.

Theorem 4.12 (Delzell and Madden). Let $A$ be a commutative unital ring. The canonical homomorphism $A \rightarrow \mathrm{F}(A)$ induces a homeomorphism between the real spectrum of $A$ and the Brumfiel spectrum of $\mathrm{F}(A)$. Hence, the Stone dual of $\operatorname{Spec}_{\mathrm{r}} A$ is $\operatorname{Id}_{\mathrm{c}}^{\mathrm{r}} \mathrm{F}(A)$. 
Say that a lattice is real-representable if it is isomorphic to the Stone dual of the real spectrum of some commutative unital ring. As in Subsection 4.2, this terminology is extended to diagrams of lattices, in a standard fashion. It follows from Theorem 4.12 that every real-representable lattice is isomorphic to $\operatorname{Id}_{\mathrm{c}}^{\mathrm{r}} A$ for some commutative unital $f$-ring $A$ (thus it is Brumfiel-representable). We will see shortly that the converse holds (cf. Corollary 4.17).

Every real-representable lattice is completely normal. By Delzell and Madden [8], not every completely normal bounded distributive lattice can be represented in this way. In fact, Mellor and Tressl established in [21] the following result.

Theorem 4.13 (Mellor and Tressl). For every infinite cardinal $\lambda$, the class of all real-representable lattices cannot be defined by any class of $\mathscr{L}_{\infty, \lambda}$-formulas of lattice theory.

The real spectrum can also be reduced to the Zariski spectrum, as follows. A commutative unital ring $A$ is real-closed (cf. Schwartz [26, 27], Prestel and Schwartz [24]) if it has no nonzero nilpotent elements, the squares in $A$ form the positive cone of a structure of $f$-ring on $A, 0 \leq a \leq b$ implies that $a^{2} \in A b$, and for every prime ideal $P$ of $A$, the quotient field $A(P)$ of $A / P$ is real closed, and $A / P$ is integrally closed in $A(P)$. Every commutative unital ring has a "real closure" $\mathrm{C}_{\mathrm{r}}(A)$, which is a real-closed ring together with a unital ring homomorphism $A \rightarrow \mathrm{C}_{\mathrm{r}}(A)$. The following result is contained in Theorem I.3.10, Propositions I.3.19 and I.3.23, and the top of page 27, in Schwartz [27].

Theorem 4.14 (Schwartz). For any commutative, unital ring $A$, the canonical homomorphism $A \rightarrow \mathrm{C}_{\mathrm{r}}(A)$ induces a homeomorphism $\operatorname{Spec}_{\mathrm{r}} \mathrm{C}_{\mathrm{r}}(A) \rightarrow \operatorname{Spec}_{\mathrm{r}} A$. Moreover, if $A$ is real-closed, then the support map $P \mapsto P \cap(-P)$ induces a homeomorphism $\operatorname{Spec}_{\mathrm{r}} A \rightarrow \operatorname{Spec} A$.

Corollary 4.15. For any commutative, unital ring $A$, the Stone dual of the real spectrum of $A$ is isomorphic to the lattice $\operatorname{Id}_{\mathrm{c}}^{\mathrm{r}} \mathrm{C}_{\mathrm{r}}(A)$ of all finitely generated radical ideals of $\mathrm{C}_{\mathrm{r}}(A)$.

Although the two following corollaries are probably well known, we could not find them explicitly stated anywhere, so we include proofs for convenience.

Corollary 4.16. Every closed subspace of a real spectrum is a real spectrum.

Proof. By Theorem 4.14, every real spectrum has the form $\operatorname{Spec} A$ for some realclosed ring $A$. By definition, any closed subspace of $\operatorname{Spec} A$ has the form $\operatorname{Spec}(A, I) \stackrel{\text { def }}{=}$ $\{P \in \operatorname{Spec} A \mid I \subseteq P\}$, for a subset $I$ of $A$, which we may assume to be a radical ideal of $A$. It follows that the assignment $P \mapsto P / I$ defines a homeomorphism from $\operatorname{Spec}(A, I)$ onto $\operatorname{Spec}(A / I)$. Now it follows from Schwartz [27, Theorem I.4.5] that the $\operatorname{ring} A / I$ is real-closed. By the second part of Theorem 4.14, it follows that $\operatorname{Spec}(A / I)$ is homeomorphic to $\operatorname{Spec}_{\mathrm{r}}(A / I)$.

Corollary 4.17. The class of real spectra of all commutative unital rings and the class of Brumfiel spectra of all commutative unital f-rings are identical.

Proof. It follows from Theorem 4.12 that every real spectrum is the Brumfiel spectrum of some commutative unital $f$-ring. Conversely, for every commutative unital $f$-ring $A$, the assignment $P \mapsto A^{+}+P$ defines a homeomorphism from $\operatorname{Spec}_{\mathrm{B}} A$ onto $\operatorname{Spec}_{\mathrm{r}}\left(A, A^{+}\right) \stackrel{\text { def }}{=}\left\{Q \in \operatorname{Spec}_{\mathrm{r}} A \mid A^{+} \subseteq Q\right\}$, with inverse the support map $Q \mapsto$ 
$Q \cap(-Q)$ (cf. Madden and Schwartz [28, page 49]). Since $\operatorname{Spec}_{\mathrm{r}}\left(A, A^{+}\right)$is, by definition, a closed subspace of $\operatorname{Spec}_{\mathrm{r}} A$, it follows form Corollary 4.16 that $\operatorname{Spec}_{\mathrm{B}} A$ is the real spectrum of some commutative unital ring.

While real-representability makes sense only for bounded lattices, Brumfiel-representability can also be defined for unbounded lattices. According to the following corollary, the two concepts agree on bounded lattices, so there is no ambiguity.

Corollary 4.18. A bounded distributive lattice is real-representable iff it is Brumfielrepresentable.

Proof. By Stone duality and Corollary 4.17, it suffices to prove that if a bounded distributive lattice is Brumfiel-representable, then it can be represented by a commutative unital $f$-ring. Let $A$ be a commutative $f$-ring such that $\operatorname{Id}_{\mathrm{c}}^{\mathrm{r}} A$ has a top element; that is, $A=\langle u\rangle_{A}^{\mathrm{r}}$ for some $u \in A^{+}$. The localization $A\left[u^{-1}\right]$ of $A$ with respect to the multiplicative subset $S \stackrel{\text { def }}{=}\left\{u^{n} \mid 0<n<\omega\right\}$, endowed with the positive cone $A\left[u^{-1}\right]^{+} \stackrel{\text { def }}{=}\left\{x / u^{n} \mid x \in A^{+}, 0<n<\omega\right\}$, is a commutative unital ${ }^{5} f$-ring, for which the canonical homomorphism $\kappa: A \rightarrow A\left[u^{-1}\right], x \mapsto x u / u$ is an $f$-ring homomorphism. Obviously, the induced map $\kappa: \operatorname{Id}_{\mathrm{c}}^{\mathrm{r}} A \rightarrow \operatorname{Id}_{\mathrm{c}}^{\mathrm{r}} A\left[u^{-1}\right]$ is surjective. We claim that $\boldsymbol{\kappa}$ is one-to-one. Let $x, y \in A^{+}$such that $\kappa\left(\langle x\rangle_{A}^{\mathrm{r}}\right) \subseteq \kappa\left(\langle y\rangle_{A}^{\mathrm{r}}\right)$. This means that $\langle\kappa(x)\rangle_{A\left[u^{-1}\right]}^{\mathrm{r}} \subseteq\langle\kappa(y)\rangle_{A\left[u^{-1}\right]}^{\mathrm{r}}$, that is, there are $z \in A^{+}$and positive integers $m, n$ such that $\kappa(x)^{m} \leq\left(z / u^{n}\right) \kappa(y)$. It follows that there are positive integers $k, l$ such that $x^{m} u^{k} \leq z y u^{l}$ within $A$. Since $\left\langle u^{k}\right\rangle_{A}^{\mathrm{r}}=\langle u\rangle_{A}^{\mathrm{r}}=A$, we obtain, using (4.4),

$$
\langle x\rangle_{A}^{\mathrm{r}}=\left\langle x^{m}\right\rangle_{A}^{\mathrm{r}}=\left\langle x^{m}\right\rangle_{A}^{\mathrm{r}} \cap\left\langle u^{k}\right\rangle_{A}^{\mathrm{r}}=\left\langle x^{m} u^{k}\right\rangle_{A}^{\mathrm{r}} \subseteq\left\langle z y u^{l}\right\rangle_{A}^{\mathrm{r}} \subseteq\langle y\rangle_{A}^{\mathrm{r}},
$$

as required. Therefore, $\operatorname{Id}_{\mathrm{c}}^{\mathrm{r}} A \cong \operatorname{Id}_{\mathrm{c}}^{\mathrm{r}} A\left[u^{-1}\right]$, with $A\left[u^{-1}\right]$ a commutative unital $f$-ring.

\section{Counterexamples COnstructed From CONDEnsates}

In the present section we shall apply the construction of a condensate, put to use in the author's paper [31] for one arrow and studied in depth in Gillibert and Wehrung [13] for more complicated diagrams. This construction enables us to construct non-representable objects from non-representable arrows (cf. Theorems 5.4 and 5.9), and it runs as follows.

Definition 5.1. Let $A$ and $B$ be universal algebras (in a given similarity type) and let $I$ be a set. The $I$-condensate of a homomorphism $\varphi: A \rightarrow B$ is the following subalgebra of $A \times B^{I}$ :

$$
\operatorname{Cond}(\varphi, I) \stackrel{\text { def }}{=}\left\{(x, y) \in A \times B^{I} \mid y_{i}=\varphi(x) \text { for all but finitely many } i\right\} .
$$

Lemma 5.2. The condensate $\operatorname{Cond}(\varphi, I)$ is a directed union of copies of algebras of the form $A \times B^{J}$ for finite $J \subseteq I$.

Proof. For each finite $J \subseteq I$, denote by $C_{J}$ the subalgebra of $\operatorname{Cond}(\varphi, I)$ consisting of all pairs $(x, y) \in A \times B^{I}$ such that $y$ is constant on $I \backslash J$, with value $\varphi(x)$. Then $\operatorname{Cond}(\varphi, I)$ is the directed union of all $C_{J}$. Clearly, $C_{J} \cong A \times B^{J}$.

\footnotetext{
${ }^{5}$ This also applies to the degenerate case where $u$ is nilpotent, in which case $A\left[u^{-1}\right]$ collapses to $\{0\}$.
} 
In particular, it follows from Lemma 5.2 that if $A$ and $B$ are members of a class $\mathcal{C}$ of algebras, closed under nonempty finite direct products and directed colimits, then so is the condensate $\operatorname{Cond}(\varphi, I)$. For example:

- Whenever $A$ and $B$ are distributive lattices with zero and $\varphi$ is a 0 -lattice homomorphism, the structure $\operatorname{Cond}(\varphi, I)$ is a distributive lattice with zero.

- Whenever $A$ and $B$ are Abelian $\ell$-groups and $\varphi$ is an $\ell$-homomorphism, the structure $\operatorname{Cond}(\varphi, I)$ is an Abelian $\ell$-group.

- Whenever $A$ and $B$ are real-closed rings and $\varphi$ is a unital ring homomorphism, the structure $\operatorname{Cond}(\varphi, I)$ is a real-closed ring.

The proof of the following lemma is an extension of the one of Wehrung [31, Theorem 9.3]. It is also an instance of a much more general result, called the Condensate Lifting Lemma (CLL), established in Gillibert and Wehrung [13], that enables to infer representability of lattice-indexed diagrams from representability of certain larger objects, called there (for the first time) condensates. In particular, as we will see shortly, Lemma 5.3 can be extended to (many) other functors than $\operatorname{Id}_{\mathrm{c}}^{\ell}$. The use of CLL requires quite an amount of technical steps and although it may be unavoidable for complicated diagrams, the case of one arrow can be handled directly; thus we include here a self-contained proof for convenience.

Lemma 5.3. Let $A$ and $B$ be distributive lattices with zero, with $A$ countable, and let $\varphi: A \rightarrow B$ be a 0-lattice homomorphism. Then for any set $I$, if the lattice homomorphism $\varphi$ is $\ell$-representable, then the distributive lattice $\operatorname{Cond}(\varphi, I)$ is $\ell$ representable. If $I$ is uncountable, then the converse holds.

Proof. Suppose first that $\varphi$ is $\ell$-representable. This means that there are Abelian $\ell$ groups $G$ and $H$, together with an $\ell$-homomorphism $f: G \rightarrow H$, such that $\operatorname{Id}_{\mathrm{c}}^{\ell} f \cong \varphi$ (as arrows). Since the functor $\operatorname{Id}_{\mathrm{c}}^{\ell}$ commutes with nonempty finite direct products and with directed colimits, it sends the representation

$$
\operatorname{Cond}(f, I)=\underset{J \subset I \text { finite }}{\lim _{\longrightarrow}}\left(G \times H^{J}\right)
$$

given by Lemma 5.2 , to a representation

$$
\operatorname{Id}_{\mathrm{c}}^{\ell} \operatorname{Cond}(f, I) \cong \lim _{J \subset I \text { finite }}\left(A \times B^{J}\right) \cong \operatorname{Cond}(\varphi, I) .
$$

Hence, $\operatorname{Cond}(\varphi, I)$ is $\ell$-representable.

Suppose, conversely, that $I$ is uncountable and the distributive lattice $E \stackrel{\text { def }}{=}$ $\operatorname{Cond}(\varphi, I)$ is $\ell$-representable. This means that there are an Abelian $\ell$-group $G$ and an isomorphism $\varepsilon: \operatorname{Id}_{\mathrm{c}}^{\ell} G \rightarrow E$. Denote by $p: E \rightarrow A$ and $q_{i}: E \rightarrow B$, for $i \in I$, the canonical projections from $E$. The set $U \stackrel{\text { def }}{=}\left\{x \in G \mid(p \circ \varepsilon)\left(\langle x\rangle_{G}^{\ell}\right)=0\right\}$ is an $\ell$-ideal of $G$; denote by $u: G \rightarrow G / U$ the canonical projection. Since $p$ is easily seen to be a closed map (cf. Definition 4.4), it follows from Lemma 4.7 that there is a unique isomorphism $\alpha: \operatorname{Id}_{\mathrm{c}}^{\ell}(G / U) \rightarrow A$ such that

$$
p \circ \varepsilon=\alpha \circ\left(\operatorname{Id}_{\mathrm{c}}^{\ell} u\right) \text {. }
$$

Likewise, for every $i \in I$, the set $V_{i} \stackrel{\text { def }}{=}\left\{x \in G \mid\left(q_{i} \circ \varepsilon\right)\left(\langle x\rangle_{G}^{\ell}\right)=0\right\}$ is an $\ell$-ideal of $G$; denote by $v_{i}: G \rightarrow G / V_{i}$ the canonical projection. As above for $U$ and $\alpha$, there is a unique isomorphism $\beta_{i}: \operatorname{Id}_{\mathrm{c}}^{\ell}\left(G / V_{i}\right) \rightarrow B$ such that

$$
q_{i} \circ \varepsilon=\beta_{i} \circ\left(\operatorname{Id}_{\mathrm{c}}^{\ell} v_{i}\right) \text {. }
$$


Since $A$ is countable, there exists a countable $\ell$-subgroup $H$ of $G$ such that $\alpha$ induces an isomorphism $\alpha^{\prime}: \operatorname{Id}_{\mathrm{c}}^{\ell}(H / U) \rightarrow A$ (where we set $H / U \stackrel{\text { def }}{=}\{x+U \mid x \in H\}$ ). We are going to argue that for every index $i$ outside a certain countable subset of $I$, the $\ell$-homomorphism $f: H / U \rightarrow G / V_{i}, x+U \mapsto x+V_{i}$ is well-defined and represents the lattice homomorphism $\varphi: A \rightarrow B$. Our argument is partly illustrated in Figure 5.1. The diagram of Figure 5.1 is not commutative, because $\varphi \circ p \neq q_{i}$ as a rule.

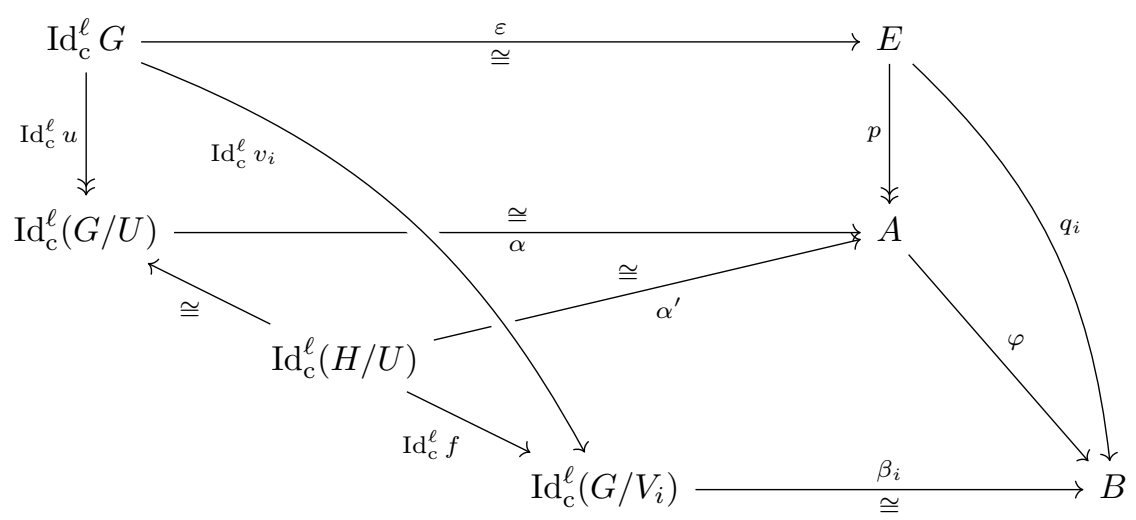

FiguRE 5.1. Illustrating the proof of Lemma 5.3

For each $x \in H$, the element $\varepsilon\left(\langle x\rangle_{G}^{\ell}\right)$ belongs to $E$, that is, $(\varphi \circ p)\left(\varepsilon\left(\langle x\rangle_{G}^{\ell}\right)\right)=$ $q_{i}\left(\varepsilon\left(\langle x\rangle_{G}^{\ell}\right)\right)$ for all but finitely many $i$. Since $H$ is countable, there exists a countable subset $J$ of $I$ such that

$$
(\varphi \circ p)\left(\varepsilon\left(\langle x\rangle_{G}^{\ell}\right)\right)=q_{i}\left(\varepsilon\left(\langle x\rangle_{G}^{\ell}\right)\right) \text { for all } x \in H \text { and all } i \in I \backslash J .
$$

Pick $i \in I \backslash J$. We claim that $H \cap U \subseteq V_{i}$. Every $x \in H \cap U$ satisfies the equations

$$
\left(\operatorname{Id}_{\mathrm{c}}^{\ell} u\right)\left(\langle x\rangle_{G}^{\ell}\right)=\langle x+U\rangle_{G / U}^{\ell}=0,
$$

thus, using (5.1), $p\left(\varepsilon\left(\langle x\rangle_{G}^{\ell}\right)\right)=\left(\alpha \circ \operatorname{Id}_{\mathrm{c}}^{\ell} u\right)\left(\langle x\rangle_{G}^{\ell}\right)=0$. By (5.3), it follows that $q_{i}\left(\varepsilon\left(\langle x\rangle_{G}^{\ell}\right)\right)=0$. By $(5.2)$, this means that $\beta_{i}\left(\left\langle x+V_{i}\right\rangle_{G / V_{i}}^{\ell}\right)=0$, that is, since $\beta_{i}$ is an isomorphism, $x \in V_{i}$, thus completing the proof of our claim.

It follows that there exists a unique $\ell$-homomorphism $f: H / U \rightarrow G / V_{i}$ such that $f(x+U)=x+V_{i}$ for every $x \in H$. For every $x \in H$,

$$
\begin{array}{rlrl}
\left(\varphi \circ \alpha^{\prime}\right)\left(\langle x+U\rangle_{H / U}^{\ell}\right) & =(\varphi \circ \alpha)\left(\langle x+U\rangle_{G / U}^{\ell}\right) & & \\
& =\left(\varphi \circ \alpha \circ \operatorname{Id}_{\mathrm{c}}^{\ell} u\right)\left(\langle x\rangle_{G}^{\ell}\right) & & \text { (use (5.1)) } \\
& =(\varphi \circ p \circ \varepsilon)\left(\langle x\rangle_{G}^{\ell}\right) & & \text { (use (5.3)) } \\
& =\left(q_{i} \circ \varepsilon\right)\left(\langle x\rangle_{G}^{\ell}\right) & & \text { (use (5.2)) } \\
& =\left(\beta_{i} \circ \operatorname{Id}_{\mathrm{c}}^{\ell} v_{i}\right)\left(\langle x\rangle_{G}^{\ell}\right) & \\
& =\beta_{i}\left(\left\langle x+V_{i}\right\rangle_{G / V_{i}}^{\ell}\right) & \\
& =\left(\beta_{i} \circ \operatorname{Id}_{\mathrm{c}}^{\ell} f\right)\left(\langle x+U\rangle_{H / U}^{\ell}\right), &
\end{array}
$$

so $\varphi \circ \alpha^{\prime}=\beta_{i} \circ \operatorname{Id}_{\mathrm{c}}^{\ell} f$. Therefore, $f$ represents $\varphi$. 
Theorem 5.4. There exists a real-closed ring, of cardinality $\aleph_{1}$, whose real spectrum is not homeomorphic to the $\ell$-spectrum of any Abelian $\ell$-group.

Proof. Let $K$ be any countable, non-Archimedean real-closed field. The subset

$$
A \stackrel{\text { def }}{=}\left\{x \in K \mid(\exists n<\omega)\left(-n \cdot 1_{K} \leq x \leq n \cdot 1_{K}\right)\right\}
$$

is an order-convex unital subring of $K$, thus it is a real-closed ring. Hence, denoting by $\varepsilon: A \hookrightarrow K$ the inclusion map, it follows from Lemma 5.2 that the condensate $R \stackrel{\text { def }}{=} \operatorname{Cond}\left(\varepsilon, \omega_{1}\right)$ is a real-closed ring. Observe that the cardinality of $R$ is $\aleph_{1}$.

Suppose that the real spectrum of $R$ is homeomorphic to the $\ell$-spectrum of an Abelian $\ell$-group $G$. By Stone duality, it follows that $\operatorname{Id}_{\mathrm{c}}^{\mathrm{r}} R \cong \operatorname{Id}_{\mathrm{c}}^{\ell} G$. Since the functor $\mathrm{Id}_{\mathrm{c}}^{\mathrm{r}}$ commutes with nonempty finite direct products and directed colimits, it follows from Lemma 5.2 that $\operatorname{Id}_{\mathrm{c}}^{\mathrm{r}} R \cong \operatorname{Cond}\left(\operatorname{Id}_{\mathrm{c}}^{\mathrm{r}} \varepsilon, \omega_{1}\right)$. In particular, the distributive lattice $\operatorname{Cond}\left(\operatorname{Id}_{\mathrm{c}}^{\mathrm{r}} \varepsilon, \omega_{1}\right)$ is $\ell$-representable. By Lemma 5.3, it follows that the lattice homomorphism $\operatorname{Id}_{\mathrm{c}}^{\mathrm{r}} \varepsilon$ is $\ell$-representable. By Lemma 4.5, it follows that the map $\varepsilon \stackrel{\text { def }}{=} \operatorname{Id}_{\mathrm{c}}^{\mathrm{r}} \varepsilon$ is closed.

However, $\operatorname{Id}_{\mathrm{c}}^{\mathrm{r}} A$ is a chain with more than two elements, $\operatorname{Id}_{\mathrm{c}}^{\mathrm{r}} K$ is the two-element chain, and $\varepsilon$ is the unique zero-separating map $\operatorname{Id}_{\mathrm{c}}^{\mathrm{r}} A \rightarrow \mathrm{Id}_{\mathrm{c}}^{\mathrm{r}} K$. In particular, if $0<u<1$ in $\operatorname{Id}_{\mathrm{c}}^{\mathrm{r}} A$, then $\varepsilon(1)=\varepsilon(u) \vee 0$ but there is no $x \in \operatorname{Id}_{\mathrm{c}}^{\mathrm{r}} A$ such that $1 \leq u \vee x$ and $\varepsilon(x) \leq 0$. Hence, $\varepsilon$ is not closed.

Remark 5.5. By (the proof of) Dickmann, Gluschankof, and Lucas [11, Proposition 1.1], the field $K$ and the ring $A$, of the proof of Theorem 5.4, can be constructed in such a way that $\operatorname{Id}_{\mathrm{c}}^{\mathrm{r}} A$ is the three-element chain. By a simple Löwenheim-Skolem type argument, $K$ may be taken countable. Then the lattice $\operatorname{Cond}\left(\operatorname{Id}_{\mathrm{c}}^{\mathrm{r}} \varepsilon, \omega_{1}\right)$ is isomorphic to the lattice $\boldsymbol{D}_{\omega_{1}}$ introduced in Wehrung [32]. As observed in the final example of Wehrung [32], $\boldsymbol{D}_{\omega_{1}}$ is a homomorphic image of an $\ell$-representable distributive lattice, without being itself $\ell$-representable. By Stone duality, this means that the spectrum of $\boldsymbol{D}_{\omega_{1}}$ can be embedded, as a spectral subspace, into the $\ell$ spectrum of an Abelian $\ell$-group, without being itself an $\ell$-spectrum.

The negative property satisfied by the counterexample $R$ of Theorem 5.4 cannot be strengthened further by replacing " $\ell$-spectrum" by "spectral subspace of an $\ell$ spectrum". The reason for this is the following easy observation, which ought to be well known but for which we could not locate a reference.

Proposition 5.6. For every commutative f-ring $A$, there are an Abelian $\ell$-group $G$, which can be taken with order-unit if $A$ is unital, together with a surjective lattice homomorphism $\mu: \operatorname{Id}_{\mathrm{c}}^{\ell} G \rightarrow \mathrm{Id}_{\mathrm{c}}^{\mathrm{r}}$ A. Hence, every Brumfiel-representable lattice is a homomorphic image of some $\ell$-representable lattice.

Proof. Denote by $G$ the underlying additive $\ell$-group of $A$. It is easy to verify that the map $\operatorname{Id}_{\mathrm{c}}^{\ell} G \rightarrow \operatorname{Id}_{\mathrm{c}}^{\mathrm{r}} A,\langle x\rangle^{\ell} \mapsto\langle x\rangle^{\mathrm{r}}$ is a well defined lattice homomorphism (use (4.3) and (4.4)). It is, of course, surjective.

Now suppose that $A$ is unital. Since the multiplicative unit 1 of $A$ may not be an order-unit of $A$, the construction of $G$ above must be modified. To this end, define $G$ as the underlying $\ell$-group of $\{x \in A \mid(\exists n<\omega)(-n \cdot 1 \leq x \leq n \cdot 1)\}$, and define again $\mu:\langle x\rangle^{\ell} \mapsto\langle x\rangle^{\mathrm{r}}$. We need to prove that $\mu$ is surjective. For every $x \in A^{+}$, the relations $x=(x \vee 1) \cdot(x \wedge 1)$ and $0 \leq x \wedge 1 \leq x$ imply that $x$ and $x \wedge 1$ generate the same $\ell$-ideal, and thus, a fortiori, $\langle x\rangle^{\mathrm{r}}=\langle x \wedge 1\rangle^{\mathrm{r}}=\mu\left(\langle x \wedge 1\rangle^{\ell}\right)$. 
By applying Stone duality to Proposition 5.6, we thus get

Corollary 5.7. The Brumfiel spectrum of any commutative unital f-ring (thus also every real spectrum) is a spectral subspace of the $\ell$-spectrum of some Abelian $\ell$-group with order-unit.

Moving to $f$-rings, a mutatis mutandis modification of the proof of Lemma 5.3, using Lemma 4.9 instead of Lemma 4.7, leads to the following result.

Lemma 5.8. Let $A$ and $B$ be distributive lattices with zero, with $A$ countable, let $\varphi: A \rightarrow B$ be a 0-lattice homomorphism, and let I be a set. If $\varphi$ is Brumfiel-representable, then the distributive lattice $\operatorname{Cond}(\varphi, I)$ is Brumfiel-representable. If I is uncountable, then the converse holds.

Theorem 5.9. There exists a real-representable distributive lattice E, of cardinality $\aleph_{1}$, with a non-Brumfiel-representable (thus non-real-representable) homomorphic image.

Proof. It follows from Dickmann, Gluschankof, and Lucas [11, Proposition 1.1] that there exists a real-closed domain $A$ with exactly three prime ideals $\{0\} \subset P_{1} \subset P_{2}$. Hence, $\operatorname{Id}_{\mathrm{c}}^{\mathrm{r}} A$ is isomorphic to the four-element chain $\mathbf{4} \stackrel{\text { def }}{=}\{0,1,2,3\}$. By an easy Löwenheim-Skolem type argument, $A$ may be taken countable. Denote by $R$ the ring of all almost constant families $\left(x_{\xi} \mid \xi<\omega_{1}\right)$ of elements of $A$. Then the lattice $E$ of all almost constant $\omega_{1}$-sequences of elements in $\mathbf{4}$ is isomorphic to $\operatorname{Id}_{\mathrm{c}}^{\mathrm{r}} R$, thus it is real-representable.

Now consider the chain $\mathbf{3} \stackrel{\text { def }}{=}\{0,1,2\}$. The map $\{1,2\} \rightarrow\{1,2,3\}$, sending 1 to 1 and 2 to 3 , is not convex in the sense of [12, Definition 13.3.10], which means that its Stone dual $\varphi: \mathbf{4} \rightarrow \mathbf{3}$ is not convex in the sense of Definition 4.10. By Lemma 4.11, $\varphi$ is not Brumfiel-representable. By Lemma 5.8, it follows that the lattice $\operatorname{Cond}\left(\varphi, \omega_{1}\right)$ is not Brumfiel-representable.

On the other hand, the assignment $\left(x_{\xi} \mid \xi<\omega_{1}\right) \mapsto\left(x_{\infty},\left(\varphi\left(x_{\xi}\right) \mid \xi<\omega_{1}\right)\right)$ defines a surjective lattice homomorphism from $E$ onto $\operatorname{Cond}\left(\varphi, \omega_{1}\right)$.

By Stone duality, it follows that the spectrum of the bounded distributive lattice $\operatorname{Cond}\left(\varphi, \omega_{1}\right)$ witnesses the following corollary.

Corollary 5.10. There exists a real spectrum with a spectral subspace which is not a Brumfiel spectrum (thus also not a real spectrum).

\section{An $\ell$-REPRESENTABLE, NON-BRUMfiel REPRESENTABLE LATtice}

Although the proof of the present section's main negative result, Theorem 6.4, arises from a lattice-theoretical investigation of the argument of Delzell and Madden [8, Lemma 2], the construction of its counterexample, which is the $\ell$-group that we will denote by $G_{\omega_{1}^{\text {op }}}$, is somehow simpler. Moreover, the constructions of Delzell and Madden [8], Mellor and Tressl [21] yield lattices with $2^{\aleph_{1}}$ elements a priori, while our construction yields the smaller size $\aleph_{1}$.

Notation 6.1. We denote by $F$ the free Abelian $\ell$-group defined by generators $a$ and $b$, subjected to the relations $a \geq 0$ and $b \geq 0$. Moreover, we set $G_{\Lambda} \stackrel{\text { def }}{=}$ $\mathbb{Z}\langle\Lambda\rangle \times_{\text {lex }} F$ (lexicographical product), for any chain $\Lambda$. 
Since $\mathbb{Z}\langle\Lambda\rangle$ is a totally ordered group and $F$ is an Abelian $\ell$-group, $G_{\Lambda}$ is also an Abelian $\ell$-group. It has an order-unit iff $\Lambda$ has a largest element. We will occasionally identify $F$ with the $\ell$-ideal $\{0\} \times F$ of $G_{\Lambda}$.

Lemma 6.2. The $\ell$-ideal $\langle a+b\rangle^{\ell}$ is directly indecomposable in the lattice $\operatorname{Id}_{\mathrm{c}}^{\ell} G_{\Lambda}$. That is, there are no nonzero $\boldsymbol{x}, \boldsymbol{y} \in \operatorname{Id}_{\mathrm{c}}^{\ell} G_{\Lambda}$ such that $\langle a+b\rangle^{\ell}=\boldsymbol{x} \vee \boldsymbol{y}$ and $\boldsymbol{x} \cap \boldsymbol{y}=\{0\}$.

Proof. Since $a, b \in F$ and $F$ is an ideal of $G_{\Lambda}$, it suffices to prove that $\langle a+b\rangle^{\ell}$ is directly indecomposable in the lattice $\operatorname{Id}_{\mathrm{c}}^{\ell} F$. The right closed upper quadrant

$$
\Omega \stackrel{\text { def }}{=}\left\{(x, y) \in \mathbb{R}^{2} \mid x \geq 0 \text { and } y \geq 0\right\}
$$

is a convex subset of $\mathbb{R}^{2}$. Further, by the Baker-Beynon duality (cf. Baker [3], Beynon [4, 5]), $F$ is isomorphic to the $\ell$-subgroup of $\mathbb{R}^{\Omega}$ generated by the canonical projections $a:(x, y) \mapsto x$ and $b:(x, y) \mapsto y$, and there exists a unique lattice embedding $\iota$ from $\operatorname{Id}_{\mathrm{c}}^{\ell} F$ to the lattice of all relative open subsets of $\Omega$ such that $\iota\left(\langle x\rangle^{\ell}\right)=\llbracket x \neq 0 \rrbracket$ whenever $x \in F$. Hence, in order to prove that $\langle a+b\rangle^{\ell}$ is indecomposable in $\operatorname{Id}_{\mathrm{c}}^{\ell} F$, it suffices to prove that $\llbracket a+b \neq 0 \rrbracket$ is a connected subset of $\mathbb{R}^{2}$. This, in turn, follows from the relation $\llbracket a+b \neq 0 \rrbracket=\{(x, y) \in \Omega \mid x+y>0\}$, which implies that $\llbracket a+b \neq 0 \rrbracket$ is a convex, thus connected, subset of $\mathbb{R}^{2}$.

Lemma 6.3. Let $\Lambda$ be a chain. Then every pairwise orthogonal subset of $G_{\Lambda}^{++}$is countable.

Proof. Since $\mathbb{Z}\langle\Lambda\rangle$ is a chain, every pairwise orthogonal subset $X$ of $G_{\Lambda}^{++}$, with more than one element, is a subset of $F$. The latter being countable, $X$ is countable.

Theorem 6.4. There are no commutative f-ring $A$ and no surjective lattice homomorphism $\mu: \operatorname{Id}_{\mathrm{c}}^{\mathrm{r}} A \rightarrow \operatorname{Id}_{\mathrm{c}}^{\ell} G_{\omega_{1}^{\mathrm{op}}}$.

Until the end of the proof of Theorem 6.4, we shall assume, by way of contradiction, that there are a commutative $f$-ring $A$ and a surjective lattice homomorphism $\mu: \operatorname{Id}_{\mathrm{c}}^{\mathrm{r}} A \rightarrow \operatorname{Id}_{\mathrm{c}}^{\ell} G_{\omega_{1}^{\mathrm{op}}}$.

Pick $x, y \in A^{+}$such that $\langle a\rangle^{\ell}=\mu\langle x\rangle^{\mathrm{r}}$ and $\langle b\rangle^{\ell}=\mu\langle y\rangle^{\mathrm{r}}$. Moreover, for each $\xi<\omega_{1}$, pick $z_{\xi} \in A^{+}$such that

$$
\left\langle c_{\xi}\right\rangle^{\ell}=\mu\left\langle z_{\xi}\right\rangle^{\mathrm{r}}
$$

In particular,

$$
\langle a+b\rangle^{\ell} \subseteq \mu\left\langle z_{\xi}\right\rangle^{\mathrm{r}}, \quad \text { whenever } \xi<\omega_{1} .
$$

Let $\xi<\eta<\omega_{1}$. Since $z_{\xi} \leq z_{\eta}+\left(z_{\xi} \backslash z_{\eta}\right)$, with $z_{\xi}, z_{\eta}$, and $z_{\xi} \backslash z_{\eta}$ all in $A^{+}$, we get

$$
\left\langle z_{\xi}\right\rangle^{\mathrm{r}} \subseteq\left\langle z_{\eta}\right\rangle^{\mathrm{r}} \vee\left\langle z_{\xi} \backslash z_{\eta}\right\rangle^{\mathrm{r}}
$$

whence, applying the lattice homomorphism $\mu$ and by (6.1),

$$
c_{\xi} \in\left\langle c_{\eta}\right\rangle^{\ell} \vee \mu\left\langle z_{\xi} \backslash z_{\eta}\right\rangle^{\mathrm{r}}
$$

Since $c_{\eta} \ll c_{\xi}$ (within $G_{\omega_{1}^{\text {op }}}$ ), it follows that

$$
c_{\xi} \in \mu\left\langle z_{\xi} \backslash z_{\eta}\right\rangle^{\mathrm{r}} .
$$

Hence we obtain, a fortiori, that

$$
\langle a+b\rangle^{\ell} \subseteq \mu\left\langle z_{\xi} \backslash z_{\eta}\right\rangle^{\mathrm{r}}, \quad \text { whenever } \xi<\eta<\omega_{1} .
$$


Lemma 6.5. For all $\xi \leq \eta<\omega_{1}$, the relation $\langle a+b\rangle^{\ell} \cap \mu\left\langle z_{\eta} \backslash z_{\xi}\right\rangle^{\mathrm{r}}=\{0\}$ holds.

Proof. The conclusion is trivial if $\xi=\eta$. Now suppose that $\xi<\eta$. We compute:

$$
\begin{array}{rlrl}
\langle a+b\rangle^{\ell} \cap \mu\left\langle z_{\eta} \backslash z_{\xi}\right\rangle^{\mathrm{r}} & \subseteq \mu\left\langle z_{\xi} \backslash z_{\eta}\right\rangle^{\mathrm{r}} \cap \mu\left\langle z_{\eta} \backslash z_{\xi}\right\rangle^{\mathrm{r}} & & \text { (use (6.3)) } \\
& =\mu\left(\left\langle z_{\xi} \backslash z_{\eta}\right\rangle^{\mathrm{r}} \cap\left\langle z_{\eta} \backslash z_{\xi}\right\rangle^{\mathrm{r}}\right) & \\
& =\mu(\{0\}) & & \text { (use (4.4) and Lemma 2.1) } \\
& =\{0\} &
\end{array}
$$

For each $\xi<\omega_{1}$, we set

$$
\begin{aligned}
& x_{\xi} \stackrel{\text { def }}{=}(x+y) \wedge\left(x z_{0} \backslash(x+y) z_{\xi+1}\right), \\
& y_{\xi} \stackrel{\text { def }}{=}(x+y) \wedge\left((x+y) z_{\xi} \backslash x z_{0}\right), \\
& \boldsymbol{x}_{\xi} \stackrel{\text { def }}{=} \mu\left\langle x_{\xi}\right\rangle^{\mathrm{r}}, \\
& \boldsymbol{y}_{\xi} \stackrel{\text { def }}{=} \mu\left\langle y_{\xi}\right\rangle^{\mathrm{r}} .
\end{aligned}
$$

Lemma 6.6. The following relations hold, whenever $\xi<\eta<\omega_{1}$ :

(1) $\boldsymbol{x}_{\xi} \subseteq\langle a\rangle^{\ell}$;

(2) $\boldsymbol{y}_{\xi} \subseteq\langle b\rangle^{\ell}$;

(3) $\langle a+b\rangle^{\ell}=\boldsymbol{x}_{\xi} \vee \boldsymbol{y}_{\xi}$;

(4) $\boldsymbol{x}_{\xi} \cap \boldsymbol{y}_{\eta}=\{0\}$.

Proof. Ad (1). From $x+y \geq 0$ and $z_{\xi} \geq 0$ it follows that $0 \leq x_{\xi} \leq x z_{0}$, whence $\left\langle x_{\xi}\right\rangle^{\mathrm{r}} \subseteq\left\langle x z_{0}\right\rangle^{\mathrm{r}} \subseteq\langle x\rangle^{\mathrm{r}}$. Apply the homomorphism $\mu$.

$A d$ (2). From (6.3) it follows that $\langle a+b\rangle \subseteq \mu\left\langle z_{0} \backslash z_{\xi}\right\rangle^{\mathrm{r}}$. Hence,

$$
\begin{aligned}
\langle a+b\rangle \cap \mu\left\langle x z_{\xi} \backslash x z_{0}\right\rangle^{\mathrm{r}} & \subseteq\langle a+b\rangle \cap \mu\left\langle z_{\xi} \backslash z_{0}\right\rangle^{\mathrm{r}} \quad \text { by Lemma } 6.5 . \\
& =\{0\}
\end{aligned}
$$

Using Lemma 2.1, we get $(x+y) z_{\xi} \backslash x z_{0} \leq y z_{\xi}+\left(x z_{\xi} \backslash x z_{0}\right)$, thus

$$
y_{\xi} \leq(x+y) \wedge\left(y z_{\xi}+\left(x z_{\xi} \backslash x z_{0}\right)\right),
$$

and thus, applying the homomorphism $\mu$ together with (6.4)-(6.5),

$$
\boldsymbol{y}_{\xi}=\mu\left\langle y_{\xi}\right\rangle^{\mathrm{r}} \subseteq \mu\left\langle(x+y) \wedge y z_{\xi}\right\rangle^{\mathrm{r}} \vee\left(\langle a+b\rangle^{\ell} \cap \mu\left\langle x z_{\xi} \backslash x z_{0}\right\rangle^{\mathrm{r}}\right) \subseteq \mu\langle y\rangle^{\mathrm{r}}=\langle b\rangle^{\ell} .
$$

Ad (3). We compute:

$$
\begin{aligned}
\langle a+b\rangle^{\ell} & =\mu\left(\langle x+y\rangle^{\mathrm{r}} \cap\left\langle z_{\xi} \backslash z_{\xi+1}\right\rangle^{\mathrm{r}}\right) & & \text { (apply (6.3)) } \\
& =\mu\left\langle(x+y)\left(z_{\xi} \backslash z_{\xi+1}\right)\right\rangle^{\mathrm{r}} & & \text { (use (4.4)) } \\
& =\mu\left\langle(x+y) z_{\xi} \backslash(x+y) z_{\xi+1}\right\rangle^{\mathrm{r}} & & \text { (use Lemma 2.1) } \\
& \subseteq \mu\left\langle(x+y) z_{\xi} \backslash x z_{0}\right\rangle^{\mathrm{r}} \vee \mu\left\langle x z_{0} \backslash(x+y) z_{\xi+1}\right\rangle^{\mathrm{r}} & & \text { (use Lemma 2.1). }
\end{aligned}
$$

Since $\langle a+b\rangle^{\ell}=\mu\langle x+y\rangle^{\mathrm{r}}$, the desired conclusion follows from the distributivity of the lattice $\operatorname{Id}_{\mathrm{c}}^{\ell} G_{\omega_{1}^{\mathrm{op}}}$.

$A d$ (4). It follows from Lemma 6.5 that $\langle a+b\rangle \cap \mu\left\langle z_{\eta} \backslash z_{\xi+1}\right\rangle^{\mathrm{r}}=\{0\}$ and hence, a fortiori, that

$$
\langle a+b\rangle \cap \mu\left\langle(x+y) z_{\eta} \backslash(x+y) z_{\xi+1}\right\rangle^{\mathrm{r}}=\{0\} .
$$


Hence, using Lemma 2.1,

$$
\begin{aligned}
\boldsymbol{y}_{\eta} & =\langle a+b\rangle \cap \mu\left\langle(x+y) z_{\eta} \backslash x z_{0}\right\rangle^{\mathrm{r}} \\
& \subseteq\langle a+b\rangle \cap\left(\mu\left\langle(x+y) z_{\eta} \backslash(x+y) z_{\xi+1}\right\rangle^{\mathrm{r}} \vee \mu\left\langle(x+y) z_{\xi+1} \backslash x z_{0}\right\rangle^{\mathrm{r}}\right) \\
& \left.=\langle a+b\rangle \cap \mu\left\langle(x+y) z_{\xi+1} \backslash x z_{0}\right\rangle^{\mathrm{r}} \quad \text { (use }(6.6)\right) .
\end{aligned}
$$

It follows that

$$
\begin{aligned}
\boldsymbol{x}_{\xi} \cap \boldsymbol{y}_{\eta} & \subseteq \mu\left\langle x z_{0} \backslash(x+y) z_{\xi+1}\right\rangle^{\mathrm{r}} \cap \mu\left\langle(x+y) z_{\xi+1} \backslash x z_{0}\right\rangle^{\mathrm{r}} \\
& =\mu(\{0\}) \quad \text { (use (4.4) and Lemma 2.1) } \\
& =\{0\} .
\end{aligned}
$$

Set $u_{\xi} \stackrel{\text { def }}{=} x_{\xi} \wedge y_{\xi}$ and $\boldsymbol{u}_{\xi} \stackrel{\text { def }}{=} \mu\left\langle u_{\xi}\right\rangle^{\mathrm{r}}$, for all $\xi<\omega_{1}$.

Lemma 6.7. The principal $\ell$-ideals $\boldsymbol{u}_{\xi}$ of $G_{\omega_{1}^{\mathrm{op}}}$, for $\xi<\omega_{1}$, are all nonzero. Moreover, they are pairwise orthogonal.

Proof. The statement of pairwise orthogonality follows from Lemma 6.6(4). If $\boldsymbol{u}_{\xi}=\{0\}$, then $\boldsymbol{x}_{\xi} \cap \boldsymbol{y}_{\xi}=\{0\}$, thus, by Lemmas 6.2 and 6.6(3), either $\boldsymbol{x}_{\xi}=\{0\}$ or $\boldsymbol{y}_{\xi}=\{0\}$, thus either $\boldsymbol{x}_{\xi}=\langle a+b\rangle^{\ell}$ or $\boldsymbol{y}_{\xi}=\langle a+b\rangle^{\ell}$, and thus, by items (1) and (2) of Lemma 6.6, either $a+b \in\langle a\rangle^{\ell}$ or $a+b \in\langle b\rangle^{\ell}$, a contradiction.

End of the proof of Theorem 6.4. By Lemma 6.7, $\operatorname{Id}_{\mathrm{c}}^{\ell} G_{\omega_{1}^{\text {op }}}$ has an uncountable, pairwise orthogonal set of nonzero ideals. Picking positive generators of the $\boldsymbol{u}_{\xi}$, we get an uncountable, pairwise orthogonal set of nonzero elements in $G_{\omega_{1}^{\text {op }}}$, in contradiction with Lemma 6.3.

As mentioned before, the real spectrum of any commutative unital ring $A$ is homeomorphic to the Brumfiel spectrum of the universal $f$-ring of $A$ (cf. Theorem 4.12). By Stone duality, we obtain the following corollary.

Corollary 6.8. The $\ell$-spectrum of the unital Abelian $\ell$-group $G_{\omega_{1}^{\mathrm{op}}}$ cannot be embedded, as a spectral subspace, into the Brumfiel spectrum of any commutative f-ring. Hence, it also cannot be embedded, as a spectral subspace, into the real spectrum of any commutative unital ring.

\section{OMitTing homomorphic IMAGES of $\ell$-REPRESENTABLE LATtices}

Although it is well known, since Delzell and Madden [8], that there are non$\ell$-representable completely normal bounded distributive lattices, the corresponding result for homomorphic images of $\ell$-representable lattices was not known until June 2017, where we posted online the first version of the present paper. In that preprint, we solved the problem negatively, by constructing a counterexample: namely, a completely normal bounded distributive lattice $D$, of cardinality $\aleph_{2}$, which is not a homomorphic image of any $\ell$-representable lattice. That structure $D$ was defined there as the lattice reduct of the free object on $\aleph_{2}$ generators, within the variety of all bounded distributive lattices (in the language $(\vee, \wedge, 0,1)$ ) with an additional binary operation $\backslash$ subjected to the identities

$$
(x \wedge y) \vee(x \backslash y)=x ; \quad(x \backslash y) \wedge(y \backslash x)=0 .
$$

The method used there, in particular the part involving infinite combinatorics (more specifically, Kuratowski's Free Set Theorem), originates in the author's paper [30] 
(living in the world of the nonstable K-theory of von Neumann regular rings). The algebraic core of that construction was contained in the commutative diagram $\vec{D}$, of bounded distributive lattices, represented in Figure 7.1, where $\mathbf{2} \stackrel{\text { def }}{=}\{0,1\}, \mathbf{3} \stackrel{\text { def }}{=}$ $\{0,1,2\}, \overline{0}=0, \overline{1}=\overline{2}=2, \boldsymbol{e}(0)=0, \boldsymbol{e}(1)=2, \boldsymbol{r}(0)=0, \boldsymbol{r}(1)=\boldsymbol{r}(2)=1$, and for all $x, y \in \mathbf{3}$,

$$
\begin{gathered}
\boldsymbol{f}(x)=(\bar{x}, x) \quad \text { and } \boldsymbol{g}(x)=(x, \bar{x}), \\
\boldsymbol{a}(x, y)=(\bar{x}, x, y, \boldsymbol{r}(y)), \\
\boldsymbol{b}(x, y)=(x, \bar{x}, y, \boldsymbol{r}(x)), \\
\boldsymbol{c}(x, y)=(x, y, \bar{y}, \boldsymbol{r}(y)) .
\end{gathered}
$$

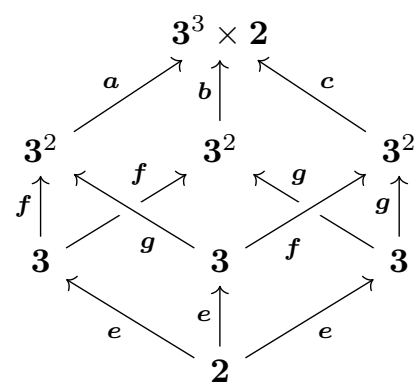

FiguRE 7.1. A cube of 0 , 1-lattice embeddings

Roughly speaking, the key property of the diagram $\vec{D}$ was that it could not be represented as the image of a cube of $\ell$-groups and $\ell$-homomorphisms under a natural transformation all of whose components are surjective $\ell$-homomorphisms.

In the meantime, the paper got lost for years in an editorial process and the above-summarized result got superseded by the author's following result, contained in [34, Corollary 7.3].

Theorem 7.1. There exists a bounded completely normal distributive lattice $B$, of cardinality $\aleph_{2}$, with no binary operation $\backslash$ satisfying the identities $\mathrm{x} \leq \mathrm{y} \vee(\mathrm{x} \backslash \mathrm{y})$, $(\mathrm{x} \backslash \mathrm{y}) \wedge(\mathrm{y} \backslash \mathrm{x})=0$, and $\mathrm{x} \backslash \mathrm{z} \leq(\mathrm{x} \backslash \mathrm{y}) \vee(\mathrm{y} \backslash \mathrm{z})$ (in [34] we call such lattices Cevian). In particular, $B$ is not a homomorphic image of $\operatorname{Id}_{\mathrm{c}}^{\ell} G$ for any representable $e^{6} \ell$-group $G$.

The lattice $B$ constructed in the proof of Theorem 7.1 satisfies one more property than the lattice $D$ above, called countably based differences and enabling the author to establish in [33], via more general variants of the condensates introduced in [13], that the class of all $\ell$-representable lattices is not closed under $\mathscr{L}_{\infty \lambda}$-elementary equivalence, for any infinite cardinal $\lambda$ (thus extending, via a completely different method, Mellor and Tressl's Theorem 4.13 to $\ell$-spectra of Abelian $\ell$-groups). This improvement forces the argument leading to the negative property of $B$, in Theorem 7.1, to be far more elaborate than the one leading to the negative property of the original $D$, in particular involving a noticeably more sophisticated variant of the diagram $\vec{D}$ of Figure 7.1 where, unlike $\boldsymbol{a}, \boldsymbol{b}, \boldsymbol{c}$ above, all the transition maps are closed homomorphisms (cf. Definition 4.4).

\footnotetext{
${ }^{6}$ Recall that an $\ell$-group is representable if it is a subdirect product of totally ordered $\ell$-groups. In particular, every Abelian $\ell$-group is representable.
} 
By Stone duality, Theorem 7.1 contains the following result.

Corollary 7.2. The spectrum $\Omega$ of $B$ is a completely normal spectral space, which cannot be embedded, as a spectral subspace, into any $\ell$-spectrum.

Final note: the countable case and the $\aleph_{1}$ case. While all containments represented in Figure 1.1 are valid for arbitrary cardinalities, the counterexamples underlying the non-containments all have cardinality $\aleph_{1}$, except for the proper containment $\mathbf{S} \boldsymbol{\ell} \varsubsetneqq \mathbf{C N}$ discussed above in this section, which involves a bounded distributive lattice of cardinality $\aleph_{2}$. This raises the obvious question about what happens for countable structures (i.e., second countable spectral spaces). The answer, provided by the author's papers [32] (for Abelian $\ell$-groups) and [35] (for real spectra), is that in the countable case the diagram collapses: that is, every second countable, completely normal spectral space is both an $\ell$-spectrum [32] and a real spectrum [35].

This leaves the following problem open:

Problem. Is every completely normal spectral space, with $\aleph_{1}$ compact open members, a spectral subspace of the $\ell$-spectrum of some Abelian $\ell$-group?

Equivalently, is every completely normal bounded distributive lattice of cardinality $\aleph_{1}$ a homomorphic image of the principal $\ell$-ideal lattice of some Abelian $\ell$-group?

\section{REFERENCES}

[1] Marlow Anderson, Lattice-ordered groups of divisibility: an expository introduction, Ordered algebraic structures (Curaçao, 1988), Math. Appl., vol. 55, Kluwer Acad. Publ., Dordrecht, 1989, pp. 3-9. MR 1094822

[2] Marlow Anderson and Todd Feil, Lattice-Ordered Groups, Reidel Texts in the Mathematical Sciences, D. Reidel Publishing Co., Dordrecht, 1988, An introduction. MR 937703 (90b:06001)

[3] Kirby A. Baker, Free vector lattices, Canad. J. Math. 20 (1968), 58-66. MR 0224524

[4] W. Meurig Beynon, Duality theorems for finitely generated vector lattices, Proc. London Math. Soc. (3) 31 (1975 part 1), 114-128. MR 0376480

[5] _ Applications of duality in the theory of finitely generated lattice-ordered abelian groups, Canad. J. Math. 29 (1977), no. 2, 243-254. MR 0437420

[6] Alain Bigard, Klaus Keimel, and Samuel Wolfenstein, Groupes et Anneaux Réticulés, Lecture Notes in Mathematics, Vol. 608, Springer-Verlag, Berlin-New York, 1977. MR 0552653 (58 \#27688)

[7] Michel Coste and Marie-Françoise Roy, La topologie du spectre réel, Ordered fields and real algebraic geometry (San Francisco, Calif., 1981), Contemp. Math., vol. 8, Amer. Math. Soc., Providence, R.I., 1982, pp. 27-59. MR 653174

[8] Charles N. Delzell and James J. Madden, A completely normal spectral space that is not a real spectrum, J. Algebra 169 (1994), no. 1, 71-77. MR 1296582

[9] — Lattice-ordered rings and semialgebraic geometry. I, Real analytic and algebraic geometry (Trento, 1992), de Gruyter, Berlin, 1995, pp. 103-129. MR 1320313

[10] Maximo A. Dickmann, Applications of model theory to real algebraic geometry. A survey, Methods in mathematical logic (Caracas, 1983), Lecture Notes in Math., vol. 1130, Springer, Berlin, 1985, pp. 76-150. MR 799038

[11] Maximo A. Dickmann, Daniel Gluschankof, and François Lucas, The order structure of the real spectrum of commutative rings, J. Algebra 229 (2000), no. 1, 175-204. MR 1765778

[12] Maximo A. Dickmann, Niels Schwartz, and Marcus Tressl, Spectral Spaces, New Mathematical Monographs, vol. 35, Cambridge University Press, Cambridge, 2019. MR 3929704

[13] Pierre Gillibert and Friedrich Wehrung, From Objects to Diagrams for Ranges of Functors, Lecture Notes in Mathematics, vol. 2029, Springer, Heidelberg, 2011. MR 2828735 (2012i:18001) 
[14] George Grätzer, Lattice Theory: Foundation, Birkhäuser/Springer Basel AG, Basel, 2011. MR 2768581 (2012f:06001)

[15] Melvin Hochster, Prime ideal structure in commutative rings, Trans. Amer. Math. Soc. 142 (1969), 43-60. MR 0251026

[16] Wolf Iberkleid, Jorge Martínez, and Warren Wm. McGovern, Conrad frames, Topology Appl. 158 (2011), no. 14, 1875-1887. MR 2823701

[17] Peter T. Johnstone, Stone Spaces, Cambridge Studies in Advanced Mathematics, vol. 3, Cambridge University Press, Cambridge, 1982. MR 698074

[18] Klaus Keimel, The representation of lattice-ordered groups and rings by sections in sheaves, (1971), 1-98. Lecture Notes in Math., Vol. 248. MR 0422107

[19] _ Some trends in lattice-ordered groups and rings, Lattice theory and its applications (Darmstadt, 1991), Res. Exp. Math., vol. 23, Heldermann, Lemgo, 1995, pp. 131-161. MR 1366870

[20] Manfred Knebusch and Claus Scheiderer, Einführung in die reelle Algebra, Vieweg Studium: Aufbaukurs Mathematik [Vieweg Studies: Mathematics Course], vol. 63, Friedr. Vieweg \& Sohn, Braunschweig, 1989. MR 1029278

[21] Timothy Mellor and Marcus Tressl, Non-axiomatizability of real spectra in $\mathscr{L}_{\infty \lambda}$, Ann. Fac. Sci. Toulouse Math. (6) 21 (2012), no. 2, 343-358. MR 2978098

[22] António A. Monteiro, L'arithmétique des filtres et les espaces topologiques, Segundo symposium sobre algunos problemas matemáticos que se están estudiando en Latino América, Julio, 1954, Centro de Cooperación Cientifica de la UNESCO para América Latina, Montevideo, Uruguay, 1954, pp. 129-162. MR 0074805

[23] Jiří Močkoř, Groups of Divisibility, Mathematics and its Applications (East European Series), D. Reidel Publishing Co., Dordrecht, 1983. MR 720862

[24] Alexander Prestel and Niels Schwartz, Model theory of real closed rings, Valuation theory and its applications, Vol. I (Saskatoon, SK, 1999), Fields Inst. Commun., vol. 32, Amer. Math. Soc., Providence, RI, 2002, pp. 261-290. MR 1928375

[25] Wolfgang Rump and Yi Chuan Yang, The essential cover and the absolute cover of a schematic space, Colloq. Math. 114 (2009), no. 1, 53-75. MR 2457279

[26] Niels Schwartz, Real closed rings, Algebra and order (Luminy-Marseille, 1984), Res. Exp. Math., vol. 14, Heldermann, Berlin, 1986, pp. 175-194. MR 891460

[27] _ The basic theory of real closed spaces, Mem. Amer. Math. Soc. 77 (1989), no. 397, viii+122. MR 953224

[28] Niels Schwartz and James J. Madden, Semi-algebraic Function Rings and Reflectors of Partially Ordered Rings, Lecture Notes in Mathematics, vol. 1712, Springer-Verlag, Berlin, 1999. MR 1719673

[29] Marshall H. Stone, Topological representations of distributive lattices and Brouwerian logics, Čas. Mat. Fys. 67 (1938), no. 1, 1-25.

[30] Friedrich Wehrung, Non-measurability properties of interpolation vector spaces, Israel J. Math. 103 (1998), 177-206. MR 1613568 (99g:06023)

[31] —, Von Neumann coordinatization is not first-order, J. Math. Log. 6 (2006), no. 1, 1-24. MR 2250951 (2007f:06011)

[32] _ Spectral spaces of countable Abelian lattice-ordered groups, Trans. Amer. Math. Soc. 371 (2019), no. 3, 2133-2158. MR 3894048

[33] _ From non-commutative diagrams to anti-elementary classes, hal-02000602, v2, preprint, 2019.

[34] Cevian operations on distributive lattices, J. Pure Appl. Algebra 224 (2020), no. 4, 106202. MR 4021916

[35] _ Real spectra and $\ell$-spectra of algebras and vector lattices over countable fields, 27 pages, June 2020.

LMno, CNRS UMR 6139, Département de Mathématiques, Université de Caen Normandie, 14032 Caen Cedex, France

E-mail address: friedrich.wehrung01@unicaen.fr

$U R L$ : https://wehrungf .users.lmno.cnrs.fr 